\title{
Italique
}

Poésie italienne de la Renaissance

IX $\mid 2006$

Varia

\section{Giovanni Guidiccioni : sonetti in sequenza d'autore (il ms. Parmense 344)}

Emilio Torchio

\section{OpenEdition}

Journals

\section{Edizione digitale}

URL: http://journals.openedition.org/italique/106

DOI: $10.4000 /$ italique.106

ISSN: $1663-4438$

\section{Editore}

Librairie Droz

\section{Edizione cartacea}

Data di pubblicazione: 1 giugno 2006

Paginazione: 29-63

ISBN: 2-600-01088-2

ISSN: 1423-3983

Notizia bibliografica digitale

Emilio Torchio, « Giovanni Guidiccioni : sonetti in sequenza d'autore (il ms. Parmense 344) », Italique [Online], IX | 2006, online dal 31 décembre 2009, consultato il 01 mai 2019. URL : http://

journals.openedition.org/italique/106 ; DOI : 10.4000/italique.106 
EMILIO TORCHIO

G I O V A N N G U I D I C C I O N I:

S ONETTI IN SEQUENZA D'A UTORE

(I L M S. P A R M E N S E 344) 



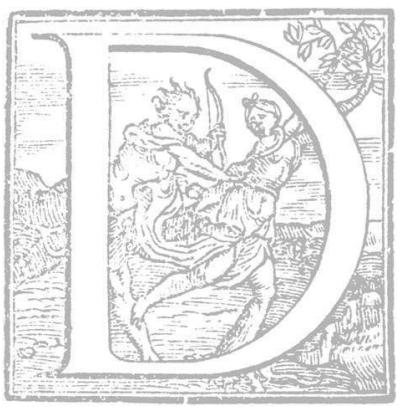

'Un qualche rilievo nella storia della lirica cinquecentesca e della forma «canzoniere» è il manoscritto Parmense 344, che monsignor Giovanni Guidiccioni allestì a Fossombrone nell'autunno del I539, durante un periodo di vacanza, bruscamente interrotto dal richiamo di Paolo III e dalla nomina a Presidente della Romagna. In esso si contengono 73 sonetti che l'autore volle trascegliere ed ordinare, dedicandoli con iniziale accompagnatoria ad Annibal Caro (c. Ir):

Messer Annibale mio, perché da l'un lato mi sento chiamare da più severo giuditio a più gravi studi, et dall'altro tirare dall'amor ch'io porto a quelle cose nelle quali mi sono affaticato, ho ridotti insieme alcuni sonetti: gli quali, desiderando per più politezza quel tempo ch'io non posso lor concedere, indirizzo così incolti come sono a voi, dalla amorevolezza et diligentia del quale non dubito punto che riceveranno più carezze, più ornamento et più lunga vita che non fariano nelle mie mane, quantunque con ogni sorte d'industria cercassi la laude et la salute loro.

Convincendo pur con qualche difficoltà monsignor Giovanni Gaddi, datore di lavoro di Caro, Guidiccioni riusci ad avere con sé il marchigiano, in qualità di segretario, per qualche mese fra la fine del' 39 e i primi del' 40 . Durante questo tempo, verosimilmente, $i$ due discussero delle rime e Caro ne propose le varianti che si possono leggere in interlinea e sui margini del Parmense.

Esso, come risulta anche ad una superficiale ispezione, non è completo: almeno due sono le carte bianche che si interpongono nella serie, altrimenti continua, di testi. Secondo un'ipotesi avanzata nell'edizione in corso di stampa per la Commissione per $i$ Testi di Lingua, alla quale si rimanda, $i$ sonetti avrebbero dovuto essere in tutto 77 e il loro ordinamento originale - scompaginato dalla legatura maldestra che a tutt'oggi resiste - può essere ricostruito grazie all'aiuto d'altri testimoni. ${ }^{\mathrm{I}}$

Il Parmense, anche dopo la morte di Guidiccioni, rimase nelle mani di Annibal Caro. Probabilmente egli ne inviò una copia a Lodovico Domenichi, che la stampò nel Libro primo delle Rime diverse, edito da Giolito nel I545. Da questo volume $i$ sonetti, arriccbiti degli acquisti apparsi nel Libro terzo del I5so (Venezia, Al segno del pozzo) e nella personale del I557 (Oratione di monsignor Guidiccione, alla Republica di Lucca, con alcune rime del medesimo, Firenze, I557), trasmigrarono in altri importanti miscellanee, fino a quel Primo volume delle rime diverse messo in commercio con frontespizi datati fra il is 86 e il 1590 (Venezia, Gioliti). Dopo 
questa data la stampa delle rime di Guidiccioni si esaurisce per più d'un secolo: il ritratto che lega Guidiccioni e Molza in un unico madrigale nella Galeria di Marino testimonia più la passata grandezza che una fama ancora attuale.

Qui di seguito si propone una ricognizione a volo radente dei sonetti del Parmense 344. Sembra utile una minuzia descrittiva perché più è alto sul testo il punto di vista più i particolari si confondono, si trascorre sulle opacità e si mettono in luce zone parziali. ${ }^{2}$ I lettori eventualmente interessati alle pagine seguenti sono di due tipi: chi si sia imbattuto, nel corso degli studi, in una rima di Guidiccioni testimoniata nel Parmense 344; chi desideri capire il funzionamento d'un macrotesto ${ }^{3}$ cinquecentesco di sicuro progetto autoriale. Ad entrambi è necessario tenere sott'occhio l'edizione che si stampa per la Commissione bolognese; in alternativa, il volume di Ezio Chiorboli per gli «Scrittori d'Italia» (Bari, Laterza, I9I2), di cui si riporta fra parentesi il numero d'ordine, può fungere alla bisogna.

Nella lettura continuata delle rime balia subito agli occhi la peculiare frattura tematica che, separando la serie in due tranches di I4 e 63 sonetti (I-I4 e I5-77), dà origine a due definiti e non amalgamati nuclei: il primo storicopolitico, il secondo amoroso-esistenziale. Non conosco soluzioni comparabili a questa. Si può ricordare, ma forse è impertinente, la scelta di Alamanni che nel primo volume delle Opere toscane (Lione, Gryphius, I532) separa i sonetti amorosi (pp. I88-257) da quelli politici (pp. 258-8I), introdotti però dalla specifica didascalia "scritti al chris. re Fran. primo». Inoltre, se la seconda tranche ha una sua strutturazione latamente "narrativa", la prima - fatta forse eccezione per $i$ suoi riguardi - non pare ordinata secondo una sequenza significativa.

Innanzৃitutto, sarà utile sgombrare il campo dall'imbarazzante, perché immeritata, fama di patriota che nel corso dei secoli si è attaccata al nome di Guidiccioni in virtu dei sonetti sul Sacco di Roma (I-I4). Rispetto a testi analoghi di Della Casa, Bembo, Molza, Cappello, B. Tasso, Tolomei, Minturno, Alamanni, Vittoria Colonna, poco varia. Guidiccioni al più si distingue per la sistemazione in gruppo o collana e per il rilievo incipitario ad essa assegnato.

[I $(I V)]$ Excelso et primo honor de gli avi tuoi. Nel primo sonetto il poeta e il Tevere, cui si dà voce, si rivolgono a Francesco Maria della Rovere per esortarlo a recare aiuto a Roma. In esso si contiene, al contrario che negli altri testi della tranche, un riferimento storico preciso, individuabile grazie a Lettere I, datata Is maggio I527: 
E a buona speranza della misericordia Sua [d'Iddio] qua è venuta novella che il duca di Urbino con le sue genti ritornava dietro, la qual cosa, se così è, a me è paruto ch'egli s'abbia lasciato fuggire una bellissima occasione di farsi glorioso per molti secoli, perciocché io porto opinione che se fusse andato avanti a soccorrere, come era conveniente, la Sua Beatitudine [Clemente VII], che averia trovate quelle genti nemiche sepolte non solamente nel sonno, ma nel vino. E di tanto popolo quanto avea quella Città, ché n'avea infinito, è quasi impossibile che non avesse trovata una parte che avesse fatta testa in qualche luogo, o che non fusse per farla al grido del suo soccorso, laonde egli combattendo potea rimendiare a molti mali e vincendo potea liberare da sì duro oltraggio colei che già fu donna di tutto il mondo. ${ }^{4}$

Gli altri testi, al contrario, fanno generico riferimento a un momento di decadenza estrema dell'Italia e di pericolo per la religione: il Sacco ne è conseguenza e, per la sua gravità, assurge a simbolo. Nulla spinge a immaginare che Guidiccioni volesse fornire un commento preciso a fatti specifici: non sembra, quindi, corretta l'operazione compiuta da Minutoli e Chiorbolis di cercare le 'occasioni' dei versi.

A parallelo del primo sonetto è utile citare Bembo LXXIX (84), I-II «Felice Imperador, ch'avanzi gli anni / Con la virtute, e rendi a questi giorni, / L'antico onor di Marte [...] / [...] / Per cui spera saldar tanti suoi danni / Roma, e fra più che mai lieti soggiorni / Sentir ancor sette suoi colli adorni / Di tuoi trionfi [...] / Mira'l Settentrion, Signor gentile: / Voce udirai, che 'n sin di là ti chiama, / Per farti sopra 'l ciel volando ir chiaro». ${ }^{6}$ Il testo, secondo la proposta dubitosa di Ugolino Martelli nella lettura di Verdeggi a l'Appennin la fronte e 'l petto (XXXVI [39]), contenuta nel Magl. VII I03 (c. 9r), sarebbe dedicato proprio a Francesco Maria della Rovere.

Il sonetto I ha un'altra specificità: è testimoniato solo dal Parmense e non dai manoscritti che recano le fasi redazionali anteriori del ciclo politico. La prima ipotesi che si affaccia alla mente è che esso sia stato composto in vista dell'organizzazione del macrotesto: a distanza d'anni, forse Guidiccioni senti il bisogno di fornire un riferimento storico un poco più esatto per togliere il velo d'appannamento steso dal tempo sulle ragioni dei suoi versi. E tuttavia non sembra probabile che Guidiccioni abbia scritto, almeno un decennio dopo $i$ fatti, il testo che più di tutti appare legato ad essi. In alternativa, bisogna supporre che il sonetto, proprio perché riferito ad avvenimenti storici precisi, sia stato tenuto segreto per ragioni di prudenza venute meno sul finire del quarto decennio del 'soo.

Gorni ha avanzato un'interpretazione d'evidente fascino: «La collana di I4 sonetti [...], se pure la serie consiste, per il numero di pezzi richiama forse le I4 stazioni della via crucis: segno, in tal caso, della perfetta fusione d'ispirazione politica e di senso religioso della storia. Roma, come Cristo, 
"Già tremar fece l'universo ad una / rivolta d'occhi, ed or cade tra via, / battuta e vinta nel suo estremo corso"》».7 Tale interpretazione, che ha il merito d'attribuire un significato al numero complessivo dei testi, coglie probabilmente nel segno, a meno di non supporre (ma è ipotesi, come si è visto, minoritaria) che la scelta d'aggiungere una quattordicesima perla alla collana infilata nel I527-28 sia tarda.

Il testo è diviso in due parti quasi uguali dal discorso diretto che il Tevere rivolge al destinatario: il periodo scavalca la divisione tra ottetto e sestetto, legando insieme seconda quartina e prima terzina. La sintassi, piana in tutto il sonetto, isola i primi quattro versi, in cui inizia il ritmo binario (Excelso / primo v. I, honor v. I / Sostegno v. 2, fido / caro v. 2, giogo / duol $v$. 3) che procede nel seguito: ardente / chiaro $v .6$, piagne / grida $v .7$, Movi $v .8$ / vien $v .9$, osi / puoi $v .8$, Fura $v . I 0$ / tinge $v$. II, sacre / gloriose v. Io (fa eccezione la terna vittorie / voglie / difetti vv. I213).

[2 (X)] Degna nutrice de le chiare genti. Il sonetto [2] è rivolto direttamente all'Italia (nutrice de le chiare genti rimanda a l'altrice de' famosi heroi $I, 4)^{8}$ e mescola rimpianto per il passato regale e dolore per il presente di servile decadenza: è strutturato su due interrogazioni, ciascuna delle quali occupa una partizione metrica, ottetto e sestetto. La sintassi procede, di conserva al sonetto precedente, su un ritmo binario: nutrice $v . I$ / albergo v. 3, genti $v . I$ / Dei v. 3, lagrime v. 4 / lamenti v. 4 , udir [...] Voci $v v .5-6 /$ mirar [...] imperio $v v$. 6-7, Tal $v .9 /$ sì v. Io, inchino $v . I I$ / adoro v. II, gloriose v. I4 / venerabil v. I4 (fa eccezione la terna pompe / pregi / impero $v v \cdot 7-8$ ).

[3 $(X I)]$ «Se pioggia homai da Dio larga non scende. In [3] il poeta riferisce all'amico d'infanzia Vincenzo Buonvisi, mercante lucchese, la propria riflessione: solo Dio, facendo piovere sul fuoco barbaro," può salvare l'Italia, che, condotta alla distruqione, non può ricevere aiuto dai suoi campioni ormai defunti. Il modulo sintattico, che dispone il discorso diretto nei $v v$. I-II e la didascalia nei vv. I2-I4, si ripresenta in 6 e in CXXXI "Com'havrà sparsi i santi odor l'Aurora (di genere pastorale di cui questa struttura è tipica); risulta invece rovesciato in $\mathrm{I}^{+} 3$ nel sonetto 9 .

[4 (VII)] Dal pigro et grave sonno, ove sepolta. Come in [2], anche in 4 il poeta si rivolge all'Italia e la invita a riscuotersi dalla servitù, cui l'hanno condotta le lotte intestine e non il valore degli avversari, in passato sconfitti. ${ }^{\circ}$ La grandezza passata è uno dei Leitmotive più evidenti e suona in ogni testo fino a 7 , per poi ricomparire a 12 e 13 . 
[s (VIII) ] Da questi acuti et dispietati strali. In s è di nuovo in primo piano l'io che vorrebbe fuggire per non dover contemplare le condizioni dell'Italia: contro di essa si accanisce con $i$ suoi strali la Fortuna, ${ }^{11}$ che apre e chiude il testo ( $v v .2$ e I3). Nei quattordici versi si snoda un unico periodo. Ben accentuato il ritmo binario: acuti / dispietati $v .1$, paventa $v .3$ / piagne $v$. 4 , alte / mortali $v .4$, levarmi $v .5$ / volar $v$. 7 , impenna / tenta $v .6$, veggia / senta $v \cdot 7$, beltà / valor $v \cdot 10$, pallida / (i)ncolta $v \cdot 10$, legge / costume $v \cdot I I$.

[6 (IX)] «Questa, che tanti secoli già stese. Analogamente a [3], nel sonetto 6 il poeta si sfoga con Buonvisi: l'Italia, un tempo vittoriosa, viene battuta dal Tedesco e dall'Ibero e non riceve aiuto dai suoi figli eminenti, Venezia e Roma. Un unico periodo si dipana nelle quartine: la prima è aperta dal soggetto ed è occupata dalle sue espansioni; la seconda dal verbo principale, che trova un coordinato al v. 7.

$[7(X I I I)]$ Prega tu meco il ciel de la su' aita. Saldo il passaggio tra Puoi tu 6, I2 e Prega tu v. I: l'identità della seconda persona non è esplicita nella redazione finale del testo e va pertanto inferita dal sonetto precedente. Il misero stato attuale dell'Italia è da addebitare all'odio intestino (cfr. L'empie tue voglie a te stessa nemiche 4, I2 e in apparato genetico le tue nemiche / Divise voglie 4, I2-I3), a disdoro di tutti e della Fortuna (come in i difetti del Fato I, I3; 5,2 e 5, I3).

[8 (XIV)] Fia mai quel dì che, 'l giogo indegno et grave. Dopo il quadro fosco del testo precedente, in 8 si sogna una condizione felice, di libertà e prosperità, ${ }^{\mathrm{I}}$ che però già si è detta d'impossibile realizzazione. ${ }^{{ }^{3}}$ Il testo è organizzato su due lunghi periodi interrogativi, che occupano l'ottetto e il sestetto, entrambi introdotti dall' anaforico Fia mai quel dì che. La sintassi, tuttavia, non è particolarmente elaborata e, come capita in 4, I-2, l'inarcatura iniziale non trova seguito.

[9 ( $)$ ] Il Tebro, l'Arno e 'l Po queste parole. Nel sonetto 9 non più il solo Tebro, come in I, ma anche l'Arno e il Po innalzano il loro lamento senza speranza, mentre l'io è in ascolto. La causa è additata nella tempesta d'occidente $v .7$, da intendersi probabilmente in modo generico 'le guerre combattute in Occidente', di cui non vengono specificati colpevoli.

$[I 0(V I)] \mathrm{Il}$ non più udito et gran publico danno. Le parole $(9, I)$ e le lacrime $(9,4)$ dei tre fiumi diventano le querele d'Italia $v v .2-3$, che suscitano il pianto nel poeta e lo susciteranno nei posteri. Come in 8, ci si 
proietta nel futuro, ma non in quello prossimo e possibile, bensi in quello lontano, quando si guarderà con occhio pietoso alle sventure causate dalla follia dei governanti e se ne cercherà una qualche vendetta.

$[I I(I)]$ Mentre in più largo et più superbo volo. Nel sonetto II si fornisce esempio del folle error evocato in I0, 9-Io: Carlo $V$, in forma d'aquila imperiale, affonda $i$ suoi artigli in Italia, disinteressandosi della Riforma che provoca la morte spirituale di quanti abitano in Germania. ${ }^{14}$ Il sonetto è d'un unico periodo, le cui partizioni sono assai evidenti, come pure il ritmo binario che lo informa: Mentre $v$. I regge la prima quartina (largo / superbo $v . I$, ali / forza $v$. 2, spande / move v. 2, altero / solo v. 4); la seconda quartina e la prima terzina si appaiano sull'anafora Non vede $v v$. 5 e 8, similmente espansa dalla relativa $\mathrm{Ch}(\mathrm{e}) v v .7$ e II (rio / perfido $v$. 5, proprio / vero $v .6$, quei $v .7$ / questi $v .8$, spavento / duolo $v .8$; danni / periglio $v .9$, verace / santa $v . ~ I 0)$; la seconda terzina si snoda a partire da Ma v. I2 (breve / vergognoso v. I3).

[I2 (II)] Ecco che move horribilmente il piede. Il rio, perfido stuolo $I I$, 5 diventa nova ingorda gente $v .3$ che cala senza trovare opposizioni in Italia: invano essa chiama aiuto, suscitando lo sdegno degli antichi condottieri vittoriosi, tra cui - per ascendenza petrarchesca - Mario. Il periodo, che si sviluppa asimmetricamente nelle quartine, è generato dall'anafora Ecco che $v v$. I $~>>$ (formula presentativa che indica l'incalzare dell'avvenimento) e, nella prima parte, procede sulle coppie move v.I / scende v. 2, Per far v.4 / Per acquistar $v .5$.

[I3 (III)] Dunque, Buonviso mio, del nostro seme. Il sonetto successivo si apre, ed è modulo non frequente, con dunque: qui indica non tanto allusione al contesto (come Bembo I28 Carlo, dunque venite a le mie rime / vago di celebrar la donna vostra [...]?), ma al cotesto, in continuazione di quanto detto precedentemente. Risulta, infatti, ben legato a I2 dal motivo dell'invasione rapinosa ${ }^{\mathrm{Is}}$ e dalla gemente preghiera (che modula le voci meste $I_{2}$, I2) rivolta dall'Italia prostrata dinnanzi a Dio, ${ }^{16}$ che con il suo intervento dourebbe restituirla al pristino stato regale.

[I4 (XII)] Vera fama fra i tuoi più cari sona. Nel sonetto I4 si prolunga, insieme ai motivi del pianto e della devastazione prodotta dagli invasori, l'interrogazione al tu, che, come indica il macrotesto e certifica l'apparato genetico, va identificato con Buonvisi, desideroso di rientrare in Italia, probabilmente da Lione a Lucca. Guidiccioni disegna un quadro cupo di morte e desolazione, una punizione infernale per i vivi e per la terra che abitano. ${ }^{17}$ Se, 
come propone Gorni, i quattordici sonetti sono le tappe della via crucis, non si profila alcuna Resurrezione. Alla prima quartina, sintatticamente svincolata, seguono dieci versi scanditi per accumulo paratattico dall'anafora di qui (modellata su Rvf. II2, 5-I3). ${ }^{\mathrm{I}}$

[Is $(X \nu)]$ Empio ver' me di sì gentil riesci. Senza soluz̧ione di continuità - nessuna carta bianca, nessun segno separatore, nessuna didascalia - dall'ultimo testo politico si passa alla sezione amorosa. Come il sonetto I, anche Is non ha carattere proemiale: non appare scritto ex post e non puì, dunque, offrire alcuno sguardo retrospettivo; non enuncia alcuna intenzione o auspicio né inscena l'inizio della storia d'amore né invoca l'aiuto di qualche divinità: siamo subito in mezzo al dolore del poeta fiaccato dalla gelosia. Della donna, a dire il vero, non ci viene detto nulla e anzi di lei compaiono appena gli occhi: solo in 30, 2 (rose vermiglie) $e$ in 32, Io (bei crin d'oro), ma in ben altro contesto, compariranno alcuni accenni fisici, topici e stilizzati al massimo consentito dalla tradizione.

I testi da I5 a I8 sono unificati dalla presenza, sempre incipitaria, di Amore (I5, 2; I6, I; I7, I; I8, I), armato del suo stral II, II; I7, 2 (e saetta al v. I3); I8, 9; del cuore dell'amante e degli occhi dell'amata. Rapporti più stretti tra loro banno Is e IG, che costituiscono un dittico. In is la gelosia trasforma l'amore prima felice in doloroso: o meglio determina una compresenza di sensazioni che sfocia comunque nel desiderio di liberarsi dall'amore. É notevole l'uso strutturale dell'antitesi: empio / gentil(e) v. I; velen v. 2 / dolce $v .3$; sicura $v .3 /$ ardito $v$. 4; dianzi mi gradisti / hor mi rincresci $v .5$; gelata cura $v .7 /$ pietà ardente $v .8$ (in chiasmo); gelo / fiamma $v . I 2$.

La vicinanza tra questo sonetto e Della Casa VIII Cura, che di timor ti nutri et cresci (Tanturli parla di «indubitabile influsso» e di «un rapporto peculiare e importante») ${ }^{19}$ è evidente e già denunciata da Minutoli ("Qual de" due abbia imitato l'altro non è si agevole da risolvere»). ${ }^{20}$ Eppure altrettanto evidente risulta essere la diversità dei due testi: a Casa importa la personificazione mitologica della gelosia che, invece, Guidiccioni ignora per concentrarsi sul soggetto sofferente. Si ricordi, inoltre, che Della Casa VI, VII, VIII formano una terna sullo stesso tema. Generici $i$ riferimenti che possono essere fatti a Sannazaro ХХVII $\mathrm{O}$ gelosia, d'amanti orribil freno (veneno v. 8, paura v. I3, Or non bastava Amor con li suoi strali? v. I4) e Cappello Is Aspro diletto, e dolce mio tormento; più stringenti quelli al più tardo sonetto di B. Tasso, IV, II (E' 'n amaro dolor giri il mio gioco v. 4; Tu col veleno tuo spargi di sorte / Ogni dolce d'amore, e rendi amaro vV. $9-10) \cdot{ }^{21}$ 
[I6 (XVII)] Mal vidi, Amor, le non più viste et tante. Evidenti i legami tra I6, testo piuttosto mediocre, e il precedente: Dovea languire $v .3 /$ deggio languir $I 5$, 9; nasce gel da le mie fiamme $v . I 0 /$ gelo in alta fiamma IS, I2; ardir $v . I 3 /$ ardito IS, 4; occhi $v .9 \mathrm{eluci} v .5 /$ occhi $I S$, I4; si ripresentano anche le antitesi: lieto 1.2 / languire $v$. 3; gel / fiamme v. Io. Il piacere provato in presenza della donna fuga $i$ sospetti del poeta, che, però, una volta lontano, è nuovamente assalito dalla gelosia e può ritrovare tranquillità solo innanzi all'amata.

[I7 (LVI)] Scaldava Amor ne' chiari amati lumi. Anche il sonetto I7 $\grave{e}$ mediocre, in particolare nelle quartine di carattere narrativo: mentre la donna piange, Amore scalda le sue frecce negli occhi di lei; il poeta, di conseguenza, mette al riparo il proprio cuore nelle lacrime dell'amata. Evidente l'inconsistenza dell'argomento: il pianto può preservare il cuore dal calore ma non dalla trafittura; secondariamente, il poeta ripara il cuore proprio laddove si trova l'arciere. Benché la sintassi sia gestita con attenzione, lasciano perplessi il susseguirsi dei tempi verbali (il presente Spingo v. 7 / consumi v. 8 è preceduto dall'imperfetto Scaldava v. I / uscian v. 4 e seguito dal perfetto Ris(e) v. I2 / Baciolli et disse v. I3), la triplice occorrenza di pietà vv. 2, 8, I4, e l'immagine finale del cuore che bacia gli occhi dell'amata ( $v \mathrm{v}$. I213).

[I8 (XVIII) $]$ Le tue promesse, Amor, come se 'n vanno. L'argomento di I 8 si articola in due momenti: nei vv. I-6 il poeta espone le lamentele contro Amore; nei successivi avanza richieste per migliorare la propria condizione d'innamorato. La sintassi, non accompagnando il contenuto, ma le suddivisioni metriche, evita giustapposizioni ofratture.

$[$ I9 $(C I X)]$ Se 'l vostro sol, che nel più ardente et vero. I successivi quattro testi (ciascuno ha schema metrico differente e nessuno il più diffuso a rime replicate) richiamano una storia d'amore un tempo felice, poi colpita dal lutto e conversa in pianto, quella fra Vittoria Colonna e Francesco Ferrante d'Avalos. Il terzetto Ig-2I è unificato dal nome di Vittoria Colonna, cui si offrono versi di consolatio per la morte del marito, avvenuta il 3 dicembre I525. ${ }^{22}$ Com'è noto, la prima a celebrare l'evento, e anzi a trasformarlo in mito personale e fondatore di poesia assegnando al consorte il senhal di «sole», fu Vittoria stessa: ${ }^{23}$ proprio da queste rime nascono, e da esse non possono prescindere, i presenti componimenti, come pure i numerosi che vari rimatori le indirizzarono intrecciando il motivo funebre, encomiastico, politico. Notevole la sintassi di I9 che lega i versi in un'unica spira. La condizione impossibile posta nella quartina iniziale, ba due apodosi. La prima incomincia con 
L'aquila $v$. 5 e ha una coordinata ai vv. 7-8 Et quel: da questo doppio corno dipende la prima terzina. Nei vv. s-II si presentano le conseguenze politiche, mentre nella seconda apodosi, che si apre con Et voi v. I2 e continua fino al termine, le conseguenze private (cui si accenna già al v. 4). Si noti che ciascuna terzina è conclusa da una similitudine (Quasi v. II, Qual v. I4).

[20 (CX)] Quanto a' begli occhi vostri et quanto manca. La prima coppia di sonetti è strettamente legata dall'uso della seconda persona plurale; dalle connessioni capfinide Quanto a' begli occhi vostri v. I / Tanto a' vostri occhi bel $I 9,4 e$ celeste corte $v .3$ / celeste sirena Iq, I4 (ma celeste ritorna anche a 2I, 6 e 22, 9); dallo svolgimento dell' argomentazione che segue una doppia via: ${ }^{24}$ il dolore intimo della moglie e il danno politico che investe tutta quanta la Cristianità, senza speranza contro il fero Scita. Il contenuto determina una struttura bipartita, che incomincia dal v. I Quanto / quanto, organizza in parallelo la zona centrale del testo Quei v. I (che richiama occhi vostri v. I) / Questi v. 9 (che richiama $i$ seguaci di Cristo v. 2), si ripercuote a chiasmo nella seconda terzina: il v. I3 pertiene al côté pubblico, il v. I4 al côté privato. Sia in Io che in 20 pare di sentire un'eco del Sacco del I527, che si propone - con tutte le cautele del caso - come terminus post quem.

$[2 I(C X I)]$ Se ben sorge talhor lieto il pensiero. Il sonetto 2I, che sarebbe arduo decifrare senza la coppia precedente, ha, invece, un tono diverso, più spirituale: una sorta d'interpretazione dell'animo della poetessa, dai legami più stretti dei due precedenti con $i$ testi di lei. ${ }^{25}$ Il tema germina dalla prima quartina di Ig (lessicalmente il v. 2, A' caldi raggi del su' amato sole, riprende sol 20, 2 e raggio 20, I2): i pensieri di Vittoria popolati dal ricordo del marito provocano dolore, mentre l'ormai impossibile presenza fisica di lui le permetterebbe d'innalzarsi spiritualmente in cielo. All'ipotesi espressa dal v. I fa da contrappunto quella del v. 8 (entrambe introdotte da Se), le cui conseguenze, giustificate nella prima terzina in cui il periodo si prolunga, sono espresse ai vv. 5-7. La seconda terzina, svincolata sintatticamente al pari della prima quartina, amplia i vv. 5-7 richiamati da Allhor v. I2.

[22(XIX)] Giovio, com'è che fra l'amaro pianto. Al gruppo si associa 22, indirizzato a un amico comune nonché intendente di poesia, Paolo Giovio. Rispetto ai testi precedenti, l'attenzione è spostata sullo specifico del canto funebre di Vittoria: della sua dolcezza, nella prima quartina, si chiede ragione a Giovio. La seconda quartina fornisce la risposta del poeta, che viene argomentata, dopo una pausa non marcata, nella prima terzina. Gli ultimi 
tre versi propongono un'altra domanda, in questo caso retorica (potrebbe anche essere intesa come esclamazione) e non più rivolta a Giovio, cui i primi versi lasciavano spazio per un sonetto in risposta.

$[23(x x)] \mathrm{Tu}$, che con gli occhi, ove i più ricchi et veri. D'improvviso, senza alcun presentimento, l'amata muore (sonetti 23-27). A livello tematico si ha una transizione per analogia, visto che la storia di Vittoria viene ad essere una sorta di prefigurazione delle vicende del poeta: come Vittoria celebrava il marito defunto cosi il poeta celebra la scomparsa della donna. Non si direbbe, quindi, involontaria la cerniera lessicale e sintattica costituita dalla rima celeste: queste 23, I0: 13, già sfruttata a 22, 9: I3 (identica la giacitura sintattica di queste in enjambement). Presagi e desideri di morte, per riunirsi all'amata nella beatitudine divina $(23,9-14),{ }^{26}$ ritornano in ciascun pezzo della serie.

Il sonetto 23 è rivolto all'amata e s'incentra sul tu anaforico ( $\mathrm{Vv} . \mathrm{I}, \mathrm{S}, \mathrm{IO})$, ribattuto da teco $v$. II $e$ te $v$. I2: il primo ampio periodo $(v v . I-8)$, strutturato dal pronome in apertura di quartine, ritarda la principale e dunque l'annuncio della morte fino al v. 7.

[24 (XXII)] Anima eletta, il cui leggiadro velo. Il leggiadro velo v. I, cioè il corpo dell' amata, ${ }^{27}$ è in antitesi a tetro mio carcer terreno di 23, 9, cielo $v .5$ richiama celeste 23, IO, angelica $v .10$ riprende angelo 23,10 . L'esortazione alla donna scomparsa, perché visiti, come quando era in vita, le notti del poeta alleviandone il dolore, è tema che richiama senz'altro Rvf. 340, soprattutto i vv. 5-7 "Già suo" tu far il mio sonno almen degno / de la tua vista, et or sostien ch'i arda / senz'alcun refrigerio», e Rvf. 250, I-3 «Solea lontana in sonno consolarme / con quella dolce angelica sua vista / madonna; or mi spaventas. La prima quartina si sviluppa dal vocativo che la inizia; la seconda si apre con la principale e prevede, in modo anomalo, una pausa di media intensità, che non interrompe il periodo, al centro del v. 6. Le terzine sono indipendenti sintatticamente, ma ben legate dalla contrapposizione, già dell'archetipo petrarchesco, tra quanto accadeva mentre la donna era in vita e quanto accade ora.

[25 (XXIII)] Come da dense nubi esce tal'hora. Il sonetto 25 ribadisce la subitanea scomparsa della donna (cfr. 23, 7 Sei spenta [...] in sì poche hore) e mostra la sua nuova dimora nella gloria dei cieli, da dove guarda e approva il poeta. Egli, compiuti gli onori funebri, attende solo la morte. Le terzine mostrano una contrapposizione temporale fra il tempo immediatamente successivo al decesso (non la vita dell'amata, come in 24) e il momento del testo, che pertanto è distanziato cronologicamente dall'evento. 
[26 (XXIV)] Poi che qui fusti la mia luce prima. L'argomento di 26 è vicino al precedente: il poeta, desiderando raggiungere l'amata che gli mostrò verità e bontà, le suggerisce le parole con cui intercedere presso Dio e garantire la probità dei desideri di lui. Nelle quartine si ha un progresso temporale: dal passato dei vv. I-4 si passa al presente dei vv. 5-6, e poi all'esortazione dell'imperativo ai $v v .7-8$; completa il movimento il futuro salirà $v .9$, che riprende, secondo l'antica connessione capfinida, salir $v .8$. Dopo una pausa non marcata, il $v .9$ accoglie la didascalia del discorso diretto a Dio dei vv. IO-I4, in cui si giustappone la condizione dell'alma v. Io del poeta e l'io vv. I3 e I4 della donna.

[27 (XXY)] Deh, vieni homai, ben nata, a darmi luce. In 27 l'amante chiede alla donna la grazia d'una sua apparizione, che sulla base di 24 si direbbe onirica: l'ottetto è scandito dai desideri rivolti all'amata vieni $v . I$, Dimmi $v .5$, Conta $v .7$, concedi $v .7$, mentre il sestetto è incentrato sulle conseguenze che essi avrebbero sull'alma del poeta: conoscenza del vero bene, consolazione, morte liberatrice. La bramosia di sapere la verità su Dio, sulla giustizia divina, sulla beatitudine germinano da 26, 7-8 Apri a l'alma i secreti di quel vero / Regno; le corone [...] et le mercedi $v .6$ richiamano merti 26, 6; l'alma, a cui già vita desti 2.9 rimodula l'incipit di 26, I-3 fusti la mia luce prima / A dimostrarmi aperto e nudo il vero, / Et festi ardenti il tepido pensiero; l'anelito al cielo dei $v v$. I2-I3 è il medesimo di 26, 8 salir in cima.

[28 (XXVIII)] A quel che fe' nel cor l'alta ferita. Il testo successivo (28) costituisce la spalla d'un ponte che, dall'altro capo, si appoggia su 6I e accoglie sotto la sua campata il secondo e maggiore ciclo amoroso del libro.

L'atteggiamento di Dante e Petrarca dinnanzi alla morte dell'amata prescriveva un'assoluta fedelta alla memoria, ad un amore che la morte aveva troncato, contro le ragioni del futuro, della ineluttabile vita ancora da vivere. Il canto si strutturava cosi in due tempi: in vita e in morte. Dante, tuttavia, aveva incontrato e descritto la sua ultima tentazione nella persona della Donna pietosa (Vita nova 24-27); Petrarca aveva forse accennato in modo criptico a un episodio simile (Rvf. 270 e $27 I$ ). Entrambi, comunque, secondo modalità distinte, avevano risolto la tentazione in nulla. In tempi contemporanei a Guidiccioni, sarà da annoverare la vicenda di Vittoria Colonna, votatasi con assoluta dedizione al marito defunto, seppure la sua condizione biografica di vedova la poneva in una prospettiva verosimilmente obbligata.

Guidiccioni compie una scelta opposta: un nuovo innamoramento, repentino e incontrastato. La nostra memoria letteraria e il nostro gusto, piu avvezzo a romanzi psicologici, rimangono perplessi. Nel poeta il nuovo amore genera, in 
principio, un senso di colpa, che spinge a invocare il perdono (28, 2). Ciononostante, la vicenda si configura subito non come traviamento momentaneo o tradimento, ma come esperienza nobilitante, dal forte contenuto spirituale e religioso. Anzi, proprio la nova fiamma 28, I2 permette all' anima del poeta, che per l'oltranza del dolore chiudeva a sé stessa il camin santo $(28$, II $)$, di tornare lietamente ad innalzarsi e a contemplare le regioni celesti. ${ }^{28}$ Rispetto alla tradizione viene, dunque, proposto un paradigma etico meno rigoroso e più praticabile. La necessità di continuare ad amare, d'appuntare, almeno nella giovinezza, i propri sentimenti verso una donna è subito coonestata sotto le ali della castità e dei pensieri santi.

[29 (XXIX)] Saglio con l'ali de' pensieri ardenti. A saldare 29 al sonetto precedente provvedono novo foco $v$. 2, che equivale a nova fiamma $28, I 2$ (e si prolunga in fiamma 30, 4), e Saglio v. I, che varia Vengo 28, I4. In esso trova ampliamento e chiarificazione quanto la giustapposizione fra le due terzine di 28 lasciava accennato: il secondo amore spinge il poeta a innalzarsi e a giungere presso l'amata d'un tempo, che di tale azione nobilitante dovrebbe essere grata alla nuova donna. ${ }^{29}$ Il ritmo binario informa il testo. A Saglio $v$. $I$, che regge le subordinate fino al v. 4, corrisponde Saglio v. , (ulteriori dualità forma et accende $v .2$ e puri et lucenti v. 5). Dopo la pausa non marcata del v. 6, il v. 7 si articola sui coordinati Quivi / quivi, cui segue al v. 8 queti et contenti. Un unico periodo si sviluppa nelle terżine: da dêi $v$. 9 dipendono Render v. II e dirle v. I2; dualità ai vv. Io e II duolo / cure $e$ gratie / salute.

[30 (XXX)] Com'esce fuor sua dolce humil favella. In 30 si esplicita che non i pregi fisici della donna, cui non si accenna, ma le parole pietose e virtuose (favella $v . I$, note $v .7$, detti $v .10$ ), ${ }^{30}$ accompagnate dai sospiri (v. 2 e v. Iz), suscitano Amore, che ragiona nella mente del poeta consigliando di smettere $i$ martiri e di cedere al nuovo sentimento (che porta con sé fiamma v. 4 , Accendi $v .6$, ardenti $v .7$, ardor $v$. I4). La circostanza che genera il testo (il profferire parola dell'amata) occupa la prima quartina; al v. , la didascalia introduce il discorso diretto, che prosegue fino al v. I4. Dopo l'esortazione del dio al poeta, vv. 6-8, seguono due interrogative, ciascuna disposta in una ter:ina, introdotte da formule parallele Non odi tu $v .9$ / Non vedi tu $v$. I2.

[3I (XXXII) $]$ O voi, che sotto l'amorose insegne. In 3 I si contiene l'invito a tutti i miliziani dell'amor virtuoso a osservare l'amata (evidente il riscontro dantesco Vita nova 2, I O voi che per la via d'Amor passate, / attendete e guardate): per lei la natura dimostra la propria potenza, 
Amore insegna la via della salvezza ed esorta a durare fatiche nel percorrerla. La seconda quartina merita d'essere riportata come esemplare della concezione d'Amore: Mirate com'Amor inspiri et regne / In sembianza del Re che 'n cielo stassi, / Come ricrei con un sol guardo i lassi / E 'l camin destro di salute insegne. Sinuosa la sintassi che prevede in apertura l'invocazione, espansa da una relativa, cui segue l'esortazione Mirate $v$. 3 replicata in anafora al $v$. 5; da essa si diparte un nuovo parallelismo anaforico $\operatorname{com}(\mathrm{e})$ v. 5 e Come 1.7 . Dopo la pausa più marcata alla fine del v. 8, segue la conseguenza Sì direte poi meco v. $9,{ }^{31}$ da cui prende le mosse il discorso diretto: due distici (esclamativo il primo, interrogativo il secondo) conclusi da un epifonema monostico.

[32 (XXXIV)] La bella et pura luce che 'n voi splende. Nel testo successivo, la luce che promana dalla donna (voi $v$. I richiama voi 3I, I, pur nella distinzione del coreferente) è Quasi imagin di Dio v. 2 (cfr. in sembianza del Re 3I, б), spinge al disprezzo degli aspetti terreni del vivere e alla virtù. Se in 3I, I2-I3 si rimpiangeva il ritardo con cui ci si è innamorati, nelle terżine di 32 si precisa che è impossibile non lodare il momento in cui l'amore è nato. Il soggetto con cui si apre il testo regge la prima quartina, cui la seconda si coordina attraverso Et iniziale del v. s. Le due terzine sono delineate nettamente da una struttura correlativa Né mi sovien $v .9$ / Ch'io non lodi v. I2, di cui è coordinata al v. I3 Et ch'io non preghi. L'ultimo verso è un'esclamazione sintatticamente svincolata, secondo un modulo che si ritrova anche a 35, I4 e 37, I4 (cfr. 36, I4, appositivo e non interiettivo).

[33 (XXXIX)] Io giuro, Amor, per la tua face eterna. La transizione da 32 a 33 è garantita da Amor v. I, strali v. 2, chiome indori v. 2 che riprendono Amor 32, I3, stral 32, I2, crin d'oro 32, IO. Il testo fornisce un catalogo di miracoli operati dalla donna, dei quali il poeta proclama la veridicità affermando d'esserne stato testimone (ho visto, da confrontare con la formula latina Vidi ego, ad es. Ovidio, Am. I 2, II). L'amata svela la propria potenza sulla natura, come in 32, sulla morte e, a seguito della trasformazione in stelle delle faville degli occhi, sugli uomini. In modo anomalo si parte dal piè per risalire al riso e poi ai lumi, procedendo in senso contrario alla topica direzione discendente della descriptio pulchritudinis. L'apostrofe all'Arno della seconda terina permette di collocare la vicenda amorosa: in quale città? La prima ipotesi è Firenze. Se diamo per scontata l'identificazione io-Guidiccioni e la pretesa verosimiglianza biografica della poesia, ci si trova però dinnanzi a un ostacolo: Guidiccioni non risulta esser vissuto a Firenze. Bisognerebbe pensare quindi a Pisa, città in cui egli fu 
studente. Entrambe le ipotesi sono compatibili con l'altra indicazione 53, I dextra riva / D'Arno, ma la prima sembra decisivamente corroborata da 42, II, in cui l'amata si appresta a tornare al natio Fiorito nido. ${ }^{32}$ L'unico periodo dei vv. I-II è vincolato al verbo iniziale giuro attraverso la triplice formula ho visto $v$. 3 , Ho visto $v$. 5 con diversa giacitura nel verso, Vist'ho v. 9 in anastrofe chiastica; la seconda e la terza occorrenza reggono la partizione metrica al cui inizio sono dislocati, mentre dalla prima dipendono i vv. $3-4$.

[34 (XL)] Dicemi 'l cor, s'avien che dal felice. I sonetti 34 , 35 e 36 formano un trittico sul cuore lontano, in possesso dell'amata. Nel primo di essi, spinto da uno scrupolo della donna, il cuore ritorna al poeta magnificando la propria vita nel petto di lei; viene, però, rinviato indietro, perché il poeta, appena caduto innamorato, aveva ricevuto il cuore dall' amata. Nella primitiva redazione riportata in apparato ( $v v .5-6)$, la dimora del cuore era collocata negli occhi di lei: pertanto ancora più evidenti risultavano $i$ legami con $i$ testi successivi (35, I-2 e 36, 5). La nuova lezione, cui si giunge verosimilmente per sostituire l'infelice v. , (al'ombra [...] della vista), genera forse una lieve incongruenza: il cuore, pur trovandosi nel petto, gode dello sguardo, dei pensieri e dei capelli di lei.

[35 $(X L I)]$ Visibilmente ne' begli occhi veggio. Precisi i rampini fra il secondo pezzo e il primo: Ivi del bel s'appaga $v .5^{33}$ richiama Godo [...] de' nei crini adorni 34, 5, Ma in disparte da lui viver m'insegna $v .7$ ribadisce Ch'è ben ragion che senza te m'acqueti 34, I4, i suoi lunghi riposi 1.9 modula per me tranquilli giorni 34, 4. Il cuore rimane negli occhi dell'amata senza intenzione di tornare presso il poeta, che, nonostante ciò, vive tranquillo. Al primo protagonista sono dedicate in prevalenza le quartine, al secondo le terzine (che si aprono, infatti, con Io).

[36 (XLII)] Sì come vola il ciel rapidamente. A funger da ponte tra secondo e terzo testo Che tornar meco ad habitar non degna 35, 6 diventa mai quindi non parte / Per unir seco il mio mortal dolente v. 8. Il lessico del sonetto è compatto: ciel vv. I e I4 (non si saprebbe dire se sia significativo questo schema ad anello); anima $v v .2$ e 7 , e la variante spirto $v$. Io (che equivalgono grosso modo a cor di 34 e 35 ); mortal $v .8$ e spoglia $v$. Io; lume $v v$. s e I3, occhi $v$. 6. Il poeta cerca di riunire il proprio corpo con la propria anima, che vive negli occhi della donna: ella, pertanto, per agevolare l'operazione non dourebbe sfuggire la sua persona. Le quartine sono collegate in un unico periodo dalla correlazione Sì come v. I / Così v. 5, che sfrutta, per il comparante della similitudine, i versi danteschi (Pd. II) sul desiderio dei 
cieli d'unirsi all'Empireo. La prima terzina presenta le conseguenze della lontananza della donna: il poeta rimane Gelato sasso che distilli humore; ${ }^{34} \mathrm{i} \mathrm{V}$. I2-I3 forniscono la logica conclusione dell'argomento: il poeta deve vivere vicino agli occhi dell'amata.

[37 (XLIII)] Falda di viva neve, che mi furi. Il sonetto 37 svolge la lode della mano: la sua contemplazione suscita diletto e $i$ detti che ba intessuto (un'impresa ricamata o una lettera) conducono all'onore. Si richiama al trittico precedente in quanto ad essa va imputato il furto del cuore. Da notare che il collegamento instaurato dai $v v .2-3$, [mano] che mi furi / Talhor il cor, risulta assente nella redazione anteriore, [mano] ch'a gli scuri / Miei torbidi pensier chiarezza rendi, e sarà, pertanto, con buona probabilità da addebitare al momento dell'organizzazione del libro. La sintassi si sviluppa attorno all'unica pausa marcata alla fine del v. 8. Grazie a un'ottima gestione dell'ipotassi, l'invocazione di v. I, espansa da subordinate, occupa la prima quartina, cui segue l'esclamazione del v. 5, che si articola nei successivi tre versi. Il legame tra ottetto e sestetto è costituito dal pronome tu $v$. 5 , ripreso in funzione strutturale nei paralleli e anaforici Tu prima $v .9 \mathrm{e} \mathrm{Tu}$ poscia $v$. I2.

[38 (XLVI) ] Sì come il sol, ch'è viva statua chiara. Il trittico successivo 38 , 39, 40 ha per soggetto gli occhi. Nel primo sonetto l'amata con il suo sguardo distribuisce a tutti luce e conforto, rendendo possibile alle menti un exitus ex se quasi mistico, l'elevazione per gradus alle regioni dello spirito e la contemplazione di Dio. Le quartine sono collegate dalla struttura correlativa Sì come v. I / Così v. s (per la quale cfr. 36, I-5). Nel suo inizzio, E 'l fa v. 9, la prima terzina mostra un forte legame argomentativo con l'ottetto; la seconda terzina riunisce in una sorta di spiegazione, o di riformulazione, i $\mathrm{vv}$. s-8 con i VV. I2-Iz, e i VV. 9-II con i VV. I3-I4.

[39 (XLVII)] Fidi specchi de l'alma, occhi lucenti. Il passaggio al secondo sonetto è garantito da occhi 38,7 (e guardo $38, I 2$ ) che ritorna in occhi $v$. I. L'incendio del cuore e la ricerca della virtù, provocati dallo sguardo dell'amata, sono qui finalizzate più al conseguimento della gloria (cfr. 50) che all'affinamento spirituale, come invece capita in 38, 5I, 52, 54. Da notare lo sfruttamento della sfera semantica della luce: lucenti $v . I$, lampi $v .2$, alluma v. 4 , raggio v. 5, Chiari v. 8, splendon $e$ faville v. 9, or v. I2. La sintassi prevede due periodi, uno nell'ottetto e uno nel sestetto. La prima quartina si apre con il vocativo, il cui sviluppo giunge al $v$. 4; la seconda con il soggetto della principale. Dal v. 9 dipendono le due consecutive Ch'io veggio vv. IO-II, Et leggio $v v$. I2-I4. 
[40 (XLVIII)] Fiamma gentil, che da' begli occhi movi. Assai vicina alla quartina iniziale di 39 è l'analoga di 40: qui si invoca la fiamma che nasce dagli occhi e riempie il cuore d'ardore, li gli occhi da cui nascono $i$ lampi che fanno avvampare il cuore. Si aggiungano lampi (39, I), arde (39, 4), raggio (39, 5) $e$ faville $(39,9)$ a far da ponte con fiamma $v$. I, faville $v .9 \mathrm{e}$ ardore $v .3$, ardendo $v .4$, arder $v .8$ (con qualche ripetizione). Lo sviluppo del tema prevede la fuga del cuore presso l'amata e la nascita d'uno nuovo nel petto dell'amante (cfr. 34-36). I vv. I-4 sono aperti dal vocativo, che si amplia attraverso subordinate; nella seconda quartina la principale è ritardata fino al v. 8 da una temporale e da consecutive coordinate. Nella seconda delle terzine, sintatticamente autonome, si notino le due esclamative di pari estensione ( $\mathrm{v} v$. I2-I3 e I3-I4).

[4I $(L)]$ Che degna schiera di pensieri eletti. In 4 I i pensieri della donna che si innalzano verso Dio aspettano e fungono da guida a quelli del poeta, che sale schernendo / Il mondo (vv. I3-I $).{ }^{35}$ Il sonetto più che al precedente (cor [...] pio 40. II e pensier pio $v .7$ ) si aggancia a 38, 9-II e, per il movimento d'ascesi, a 39, 9-II; ma soprattutto mostra consonanze con 54, 9-II veggio di lontan faville / Che le più folte oscure nebbie aprendo / Segnano il bel sentier ch'al ciel aggiunge, cui segue, nell'ultima terżina, una similitudine con la stella cadente, assai vicina a quella dei vv. I2-I4 Stella nel nascer suo del mare schiva. Anche se nella lettura è arduo mantenere l'intonazione adatta, le quartine sembrano accogliere un unico periodo esclamativo, in cui si move et va volando $v .3$ è coordinato a par $v .5$, da cui dipendono aspetti $v$. 5 e preghi $v .6$.

[42 $(L I)]$ Chi desia di veder dove s'adora. Nel sonetto 42 si rappresenta una vicenda geograficamente precisa. La donna si trova a Roma: il poeta invita ad affrettarsi chi voglia vedere ${ }^{36}$ un miracol sì novo, quasi fosse l'ostensione d'una reliquia, perché è prossimo il ritorno di lei a Firenze, sua città natale. Un unico periodo riccamente ipotattico si sviluppa ai vv. I-II. Come desia $v$. I regge la prima quartina, così verbi principali venga $v$. 5 e indugi $v .9$ reggono rispettivamente la seconda quartina e la prima terzina: la loro posizione incipitaria conferisce ordine e agevola la lettura. La seconda terzina si inižia con Vedrà, che riprende veder $v$. I e mirar $v$. 5. Da osservare il ritmo binario: dove $v . I$ e v. 3 ; che $v .5 /$ a cui $v$. 7 ; come v. I2 / quanto v. I3.

[43 (LII)] Sovra un bel verde cespo, in mezzo un prato. Il sonetto successivo risulta dissonante allinterno del segmento macrotestuale e della silloge intera. Appartiene al genere «bucolico-pastorale» e alla specie «sacrificios: esibisce, del primo, il topico accenno all'amore omosessuale, l'ambienta- 
zione, il nome Tirsi, la scelta di Zephiro, le accensioni coloristiche; del secondo, la presenza dell'altare e delle vittime, dei voti e degli incensi. Chi dice io offrirà il proprio tributo al vento se esso rinfrescherà l'amata. Il tema è classico, non dantesco o petrarchesco: ad es. cfr. Tibullo I I, 23-24 «agna cadet vobis, quam circum rustica pubes / clamet "io, messes et bona vina date!"”, I I0, 26-28 «hostiaque e plena rustica porcus hara. I Hanc pura cum veste sequar myrtoque canistra / vincta geram, myrto vinctus ipse caput); nei sacrifici la presenza delle vittime si accompagna sovente a quella di giovinette e giovinetti, cfr. Ovidio, Am. III I3, 24 «invenes timidaeque puellae» (Gorni ricorda Carmen saeculare 6 «virgines lectas puerosque castos»). ${ }^{37}$ Per trovare prove simili dobbiamo volgerci ad alcune rime non raccolte nel Parmense, Dimmi, se il tuo desio come esser suole $(L X X X V)$, Eran pur dianzi qui tra le fresche herbe $(L X X X V I)$, Com'havrà sparsi i santi odor l'Aurora (CXXXI), ma soprattutto l'inedito Zephiro, eterna primavera infiora $(C X X I)$, che è preghiera al vento di stretta somiglianza col presente testo. Notevole la sintassi che avvolge il sonetto in un'unica spira, tanto più che il punto di pausa più marcata cade non in fine di verso o di partizione metrica, ma a metà del v. s. Da lì iniżia una tripartizione tematica e strutturale (da l'un lato / Donne $v v$. 5-6, Dall'altro [...] giovani v. 9, tutti $v . \quad I 0)$ conclusa, nell'ultima terzina, dalla condizione in base alla quale quanto prima descritto si verificherà.

[44 (LIII)] Vedrà la gente homai che quanto dissi. Non desta sorprese che il sonetto 44 si leghi non al precedente ma a 42, di cui replica in incipit Vedrà 42, I2. Da 44 a 48, e poi a 52, in ogni pezzo ritorna la castità della donna; allargando l'attenzione all'honestà il gruppo si amplia, in un senso, a 42, nell'altro fino a 53. Anche a questo tema si interseca la presenza dell'Arno (cfr. 47, 9 e 49, I). Ne risulta una porzione compatta, con l'anomala presenza di 43: sottilizzando, ma senza convinzione, si può trovare qualche somiglianza tonale, visto il comune décor mitologico, fra 43, I-6 e 46, I-8, in cui si rammenta il pianto di Venere sul corpo di Adone.

Il sonetto proclama la veridicità di quanto affermato finora sulla virtù dell'amata, superiore alla Lucrezia liviana (ma più che Livio I, 57-60 varranno Rvf. 260, 9-Io e Tr. Pud. I30-I32), e la felicità di chi la ama. I vv. I-6 sono costruiti sull'anafora di Vedrà $v$. I e v. 5 , cui si coordina dirà $v .6$ che introduce la seconda parte, vv. 7-I4, occupata dal discorso diretto. Dopo un distico esclamativo, centro geometrico e tematico del componimento, un unico periodo prosegue senza ambagi fino al termine.

[45 $(L I V)] \mathrm{O}$ cor più ch'altro saggio et più pudico. Evidenti $i$ legami di transizione fra 44 e 45: nemico 44, I3 e v. 4 (tema che ritorna a 48, 5-6), 
nell'età ch'ella fioriva 44, Io e fiore / De gli anni $v$. 3-4, casta 44, I2 $e$ castità v. I4. La saggezza e la pudicizia della donna che ha sconfitto il demonio dourebbero essere celebrati con sfarzo nel mondo. I vv. 9-I4 mi pare presuppongano una diceria corsa sull'onestà della donna, che ne piange (cfr. 46, 9-II e 53: un accenno all'invidia in 36, I2-I3 apparato, ai detrattori o maligni 38, II e I2-I3 apparato): ma la castità stessa difende l'innocente, cosi che ella se ne debba consolare. Il testo è formato da due periodi che occupano le macropartizioni di ottetto e sestetto. Nella prima quartina è il vocativo e le sue espansioni, nella seconda due principali coordinate esclamative costruite sui paralleli Se v. , / Et se v. 7. La prima terzina si apre con la principale cui fanno seguito due coordinate relative; nella seconda terzina due causali coordinate, dalla seconda delle quali dipendono altre due coordinate.

[46 (LV)] Sovra 'l bel morto Adon non fur già quelle. Il pianto di 45, 9-II e le dicerie adombrate a 45, I2-I4 diventano tema di 46, la cui organizzazione è assai precisa. Nella prima quartina trova posto la descrizione di Venere che piange Adone; ma la donna è superiore alla dea: nella seconda quartina e prima terzina se ne descrive il pianto, nella seconda terzina le parole (corrispondenti ai sospiri della dea), che dichiarano al poeta la speranza di non essere infamata e, forse, il proprio amore per lui. ${ }^{38}$ Un unico periodo avvolge i primi II versi. Le quartine sono legate da un rapporto comparativo sì $v .2 / \operatorname{così~} v .3$, Come $v .5$; da vid'io $v .5$ dipendono $i$ due coordinati Sparger $v .6$, che regge la seconda quartina, e mover $v .9$, che regge la prima terzina. Gli ultimi tre versi sono occupati dal discorso diretto e dalla sua didascalia, svincolata sintatticamente dai versi precedenti.

[47 (LVII)] Donna, che 'ntesa a bei pensier d'honore. Il sonetto 46 e il successivo sono collegati da Stral 46, 13 / stral $v .6$ e casti $46, I_{4} /$ castità $v$. 2. Il riconoscimento altrui del valore della donna (vv. 9-I4) stabilisce un legame con in memoria del tuo bel valore / [il mondo] Pianti mill'alte palme 45, 7-8. La castità e la bellezza in grado eminente, la capacità di resistere ad Amore (in contrasto con quanto sembra affermato a 46) costituiscono i pregi dell'amata, che le frutteranno onori da parte dei fiorentini; $i$ quali stigmatizzeranno, poi, l'insufficienza del canto del poeta. La prima quartina si apre con l'invocazione e contiene le espansioni che da essa dipendono; la seconda quartina si inizia con la principale e ospita un paio di subordinate. Ciascuna terzina è invece autonoma; la seconda è retta dall'iniziale Diran, da cui dipendono due oggettive (che $v v .12$ e 13 ).

[48 (LVIII) $]$ Fonte d'alto valor, de' cui bei rivi. Il passaggio tra 47 e 48 avviene «sull'acqua» (fonte $e$ rivi $v . I$ richiamano il mare 47, I2), ma è 
rafforzato dall'ambientazione fiorentina (47, 9 e v. 2), dal comune tema del canto del poeta (47, I3 e v. I3) e della castità (47, 8 e v. 4). La castità risulta qui minacciata da persone serbate vive da Dio per aumentare la gloria della donna, non dal metafisico Demonio (44, I4 e 45, 5). La donna è destinata perciò all'immortalità, ma, intanto, è sia desiderata dagli spiriti del paradiso sia invocata, alla stregua d'una santa, nelle preghiere dei vivi; per il poeta svolge la stessa funzione dello Spirito Santo nella Pentecoste: ne infiamma lo stile. Un unico periodo si snoda nei quattordici versi, con una pausa più marcata in punta di v. I2. Fino al v. 6 domina l'ipotassi, in dipendenza del vocativo con cui si apre il componimento; dal $v .7$ in avanti prevale, al contrario, la paratassi.

[49 (LIX)] Spargete, o nimphe d'Arno, arabi odori. In 49 sembra compiuto quanto preannunciato in 42: la partenza dell'amata da Roma per la città natale. Più precisamente, nel sonetto si descrive il momento in cui la donna appare in vista della città natale e il poeta invita a far festa per il suo ritorno, la sua castità, la sua vita esemplare, le cui celesti virtù donano pace e salvazione. Evidenti le somiglianze con la domenica delle palme (citate come premio a lei dovuto, insieme agli allori, al v. 8), ma qui il décor è tenuemente mitologico (Nimphe v. I e Amori v. 5). ${ }^{39}$ Tutto il sonetto invera 47, 9-II $\mathrm{O}$ qual da' saggi et chiari figli d'Arno / Corona di topatii et di diamanti / Vi si prepara et quai triomphi et archi: forse da rilevare il ricordo del modulo evangelico per cui si annuncia un fatto e poi si evidenzia il suo compimento (cfr. Mattheus 2, I7 «Tunc adimpletum est quod dictum est per Ieremiam prophetam»). Sotto il largo dominio della paratassi, la prima quartina contiene l'esortazione alle ninfe, la seconda quartina agli Amori, il cui canto di gioia per la donna occupa la seconda parte del testo vv. 7-I4.

[5o (LX)] A la bell'ombra dela nobil pianta. L'onestà 49, $7 / v .2 e$ l'alloro 49, 8 / v. I agevolano il passaggio al testo successivo, che costituisce una sorta di pausa allinterno della vicenda principale. E testo in lode della poesia: se ne stabilisce recisamente il nesso inscindibile con le virtù etiche dirette alla trascendenza, nesso simboleggiato dall'alloro grazie al mito dafneo sotteso. A seguito della condotta ispirata dalla nobil pianta, il poeta ha sconfitto $i$ vizi conseguendo la gloria; spera, pertanto, d'essere incoronato e d'ascendere a regioni celeste non mai prima raggiunte da alcuno.

[SI $(L X I)]$ Gratie rendo a' bei lumi honesti et chiari. Il componimento successivo, il cui contenuto si affastella slegato, è un ringraziamento alla donna: il suo sguardo ba provocato l'accensione nobilitante del cuore che permette di sconfiggere gli errori (cfr. possenti desir 50, 5, Schiere de' vitii 
50, 6) e d'aspirare alla gloria (cfr. le tempie ornate / Spero anco haver de la sua fronde santa 50, 7-8). Notevole è la presenza della sfera semantica della luce (lumi, rai, soli, lume) e del fuoco (accese, avampa, ardore, fiamma).

[52 (LXII)] Splende nel mio pensier l'imagin viva. A differenza del precedente, 52 ha un'argomentazione ordinata e mostra legami precisi con esso: Splende nel mio pensier l'imagin viva ( $(v . I)$ è conseguenza di sent'io scolpir ne l'alma / Le vere forme ( $I, 9-I O)$; lei che m'arse 'l cor $(v .2) \grave{e}$ analogo a mosse vertù ch'accese il core $(5 I, 2)$; salissi $v .2$ rimanda, poi, a Per non trito sentier salir in parte 50, 10. Anche il solo ricordo visivo e uditivo dell'amata spinge all'elevazione spirituale, cosi che il poeta si può nutrire del vero bene senza essere funestato dalla forza dell'oblio; dal ricordo lo spirito deriva, poi, l'ispirazione per la poesia. Probabilmente da respingere la tentazione di vedere nei $v v$. I2-I3 nel suo casto seno / Vola audace talhor lo spirto mio un rimando al trittico $34-36$.

[53 (LXIV)] Parmi veder che su la dextra riva. In s3 la donna, non vedendo difesa dall'amante la propria onorabilità messa in dubbio dalle insinuazioni del volgo, esprime il timore per la morte della propria fama e il rammarico per l'amore sfiorito del poeta. Se l'interpretazione proposta per 45, 9-I4 e 46 è corretta, questo testo si riferirebbe allo stesso (o consimile) episodio: in entrambi si ritrova la tristezza dell' amata e una diceria malevola. Il $v$. II Ond'hebbe lume la sua oscura vita costituisce un rampino all'indietro: cfr. SI, 8 Onde l'oscuro de' miei dì rischiari. Il testo è bipartito tra ottetto e sestetto, parallelamente introdotti da Parmi veder $v .8$ e Parmi sentir v. 9 . Le quartine sono occupate da un solo periodo d'ipotassi pregevole, in cui Parmi veder regge $i$ coordinati s'assida $v$. I e Et [...] s'attriste $v v$. $5-7$.

[54 $(L X V)] \mathrm{Al}$ chiaro foco del mio vivo sole. Il sonetto 54 si riallaccia non al precedente, ma per $i$ versi iniziali $\mathrm{Al}$ chiaro foco del mio vivo sole, / Ov'accende virtù suoi caldi raggi $a$ SI, $I-2$ Gratie rendo a' bei lumi honesti et chiari, / Onde mosse vertù ch'accese il core; per il v. 4 Col pensier miro sue bellezze sole $a$ 52, I Splende nel mio pensier l'imagin viva; e per l'ascensione al cielo dei vv. 7-8 [l'alma] Ferma di gir per dritti alti viaggi / Al'eterno Signor che sembra et cole $a$ 50, 9-Io E con lei poi, che dritta s'erge al cielo, / [...] salir in parte (luogo che ha, però, connotati meno evidentemente religiosi). Il poeta, pur trovandosi lontano dall'amata in un ambiente bucolico (cui sia accenna di scorcio al v. 3), riesce a supplire alla distanza col pensiero e a contemplarne le bellezze; desideroso d'alzarsi al cielo, scorge la traccia luminosa, come di stella 
cadente, lasciata dalla donna e la segue. La sintassi e l'argomento sono ben legati: Qui v. 5 replica in parallelo qui $v .3$, sentier $v$. II si lega ad alti viaggi $v .7$, lunge $v .13$ riprende in diverso contesto di lontan $v .9$.

[s) (LXXIV)] Qui dove i lumi bei solean far giorno. Il gruppo che si estende fra 55 e 60 è formato da testi di corrispondenza: dopo i sonetti politici, il cui interlocutore era Vincenzo Buonviso, e il gruppo 19-22, in cui ci si riferiva a Vittoria Colonna e Paolo Giovio, sono i primi in cui faccia irruzione un personaggio estraneo alle vicende amorose.

Il sonetto iniziale non ha, a differenza dei seguenti, un destinatario nominato. In virtù della vicinanza contenutistica col testo successivo, dedicato a Girolamo da Correggio, si può supporre che formi con esso un dittico. Il sonetto si lega al precedente per il tema della dell'amata lontana e per l'anafora di qui $v v .1-2 e$ 54, 3-5. A quanto pare (e non è situazione lirica diffusa), il poeta e il dedicatario sono innamorati della stessa donna (tue notti et mie $v .2$, N'aperse v. 3), ma nutrono ciascuno un amore diverso: spirituale il primo, cosi che riesce a supplire col pensiero all'assenza di lei (cfr. 52 e soprattutto 54, ${ }_{4} \mathrm{Col}$ pensier miro sue bellezze sole) e pregia l'anima più che il corpo; men pudico il secondo, legato alla presenza fisica dell'amata. Dal punto di vista strutturale, la prima quartina è dedicata alla proposizione del tema, la seconda al poeta; le teriine, invece, al destinatario.

[56 (LXIII)] Correggio, se 'l tuo cor sospira invano. Il sonetto s6 condivide con il precedente il tema della non necessaria vicinanza dell'amata e il confronto tra la situazione amorosa del destinatario e del poeta. La disposižione della materia è chiastica rispetto a 5s: i primi quattro versi dedicati a Correggio, i restanti al poeta, che - spingendo la propria mente a contemplare l'anima della donna - non teme né l'invidia altrui né il fiele d'Amore. Si propone qui una soluzione al problema della sofferenza d'amore, causata dalla presenza d'altre persone a contorno della storia privata degli innamorati: il solo amore inattaccabile dalle dicerie e dalla gelosia è la comunione tra anime rivolte a un bene metafisico. Le prime due quartine sono collegate sintatticamente attraverso una struttura oppositiva se 'l tuo cor $v$. I, Io $v$. I (cfr. Is, $9 \mathrm{Tu}$ in analoga posizione forte). La seconda terzina inizia con Né tem'io che da un lato riprende Io v. s e dall'altro prepara Né v. II.

[58 (CXIII)] Scipio, io fui rapto dal cantar celeste. Segue un ulteriore, $e$ più indubitabilmente delineato, dittico. La prima quartina di 58 è divisa in distici: il primo enuncia il rapimento estatico determinato da un canto forse religioso, il secondo la riflessione che ne segue. Tra questi due momenti cronologici si colloca la descrizione della cantante fornita dai versi restanti, in cui i verbi coordinati sono coningati allimperfetto, il tempo dei sogni raccontati. 
[59 (LXVI)] Questo, che gli occhi abbaglia et l'alma accende. Il secondo testo continua l'argomento del precedente (divina / Sua voce $v v$. 9-Io si collega a cantar celeste 58, I, rapina $v$. II a rapto 58, I voci $v . I 2$ a suon 58,7$)$ : la bellezza corporea del riso e degli occhi rasserena il dolore ( $v v$. I-4) e purifica (vv. 5-8); ma è la voce impalpabile che esalta e spinge all'ascesi (vv. 9-I I ) ${ }^{40}$ Il testo, si badi, costituisce un'eccezione rispetto al gruppo che si è individuato perché contiene non l'allocuzione diretta al destinatario, ma solo mediata dal testo precedente.

[60 (XCI)] Mentre che voi, cui vien dal ciel concesso. Nel sonetto 60, indirizzato a un personaggio ignoto, militare e letterato che fugge dall'amore per dedicarsi alla spiritualità, la descrizione che il poeta fa di sé come un innamorato dubitoso e sofferente (vv. 9-I4), in cerca d'uno sguardo per avere requie, è poco consona a questa zona macrotestuale. Il topico confronto tra la situazione del destinatario e quella dellio determina la struttura bipartita del testo, in cui l'ottetto è assegnato al primo e il sestetto al secondo. La sintassi, però, non si adagia sulla suddivisione tematica: il primo periodo, assai elaborato (la doppia finale Per honorar $v .3$ / per ornar $v$. s e la doppia relativa si consiglia $v .9$ / nudre $v$. Io sono entrambe anticipate rispetto alla reggente), abbraccia i $\mathrm{Vv} \cdot \mathrm{I}-\mathrm{II}$, il secondo i $\mathrm{Vv}$. I2-I4.

[6I (XXXVI)] Lo stral, che 'n sorte hebb'io, dentr' a' begli occhi. Al pari del precedente, la posizione di 6 I all'interno della silloge è poco comprensibile: vi si descrive, infatti, l'innamoramento del poeta. Gli attori della vicenda, che ha una forma narrativa e dialogica, sono solo in parte quelli tradizionali: il ruolo della donna è impersonato, con uno slittamento metonimico, dal sentimento di pietà da lei nutrito nei confronti del poeta, che Amore sta per trafiggere. E la Pietà ad essere il riferimento grammaticale costante dei pronomi (lei $v .7$, Le $v .9$ ), ma la semantica dei verbi (dicea $v .6$, piacer bevea $v .7$, arrise et disse v. 9) è più adeguata alla donna. I testi che, da I4 in poi, citano la pietà - I7 (con cui il presente ha rapporti privilegiati, benché quello sia riferito al primo amore) 30, 37 e 40 - non pare diano a questo sentimento un rilievo esplicito, né rispetto ad essi pare che esista un'evoluzione o un mutamento ideologico. Forse (ma la spiegazione è poco persuasiva) mettendo in risalto il ruolo positivo della Pietà quasi a scapito di Amore (Dolce piaga vital [...] Viva ti tien Pietà via più ch'Amore vv. I2-I4), e la gioia e la virtù generate da esso, il poeta vuole dare una sorta di gindizio ultimo sulla vicenda amorosa, marcandone il carattere fondamentale. La sintassi non è limpida e necessita di particolare attenzione per essere dipanata, specie a causa dei continui cambi di soggetto. 
[62 (LXIX)] Avvezzianci a morir, se proprio è morte. Il sonetto 62 rappresenta la svolta con cui si entra nella zona conclusiva, il cui tono è dapprima morale e spirituale, poi sempre più religioso. Il testo è un'esortazione alla mediatio mortis $e$ alla «morte nella vita» come unico mezzo per giungere all'elevazione dello spirito: nessuna altra condotta di vita va approvata e seguita. L'anima ha legami eccessivamente forti con il corpo e la bellezza transeunte della realtà per potersi innalzare sopra il sensibile, ${ }^{4 \mathrm{I}} e$ dimentica cosi la sua origine divina. Si rifiutano, pertanto, tutte quelle vie e quelle pratiche di vita, collegate all'amore per una donna, che innalzano al cielo ed esortano alla virtù (cfr. per contrasto Dolce piaga vital, c'hor sì verace / Gioia distilli et crei virtù diverse 6I, I2-I3). Sintatticamente il testo si compone di due periodi: il primo (vv. I-II) è assai ampio, pur basandosi su una principale minima Avvezzianci v. I, e si sviluppa sulla reduplicatio L'alma $v .3$ / L'alma $v$. s, che regge due relative $\operatorname{ch}(\mathrm{e}) v .5$ / Che v. 9 .

[63 (CXVIII) $]$ Crispo, se avvolto sei tra scogli et sirti. I successivi testi formano un trittico di corrispondenza dal contenuto morale, d'una moralità in buona sostanza oraziana: l'accenno agli splendor celesti di 64, 6 potrebbe essere sottoscritto anche da un platonico senza Rivelazione. Risulta piuttosto scabro il passaggio tra 62, che propone una visione cristiana quasi ascetica, $e$ questi temi, elevati ma essenzialmente «laici».

Verosimilmente, il primo sonetto è indirizzato a Tiberio Crispo, cardinale nel I544. Dal v. s si deduce che Crispo aveva confessato le proprie ambizioni e inquietudini, che resistevano a dispetto dell'età matura. Il poeta replica con parole amichevoli, disposte secondo una sintassi paratattica e scorrevole, da colloquio colto, non da risposta a una quaestio morale: solo i desideri limitati trovano soddisfazione $e$ il tempo è tanto veloce a fuggire quanto $i$ danni a giungere. Da notare come al tricolon del $v .7$ rispondano la triplice anafora di come $(v v .9$, Io e II) e le tre coordinate dei vv. I2-I4.

[64 (CI)] Chi per quest'ombre de l'humana vita. La navigazione notturna in mari insidiosi di 63, I-2 diventa, nel sonetto successivo, un percorso pieno d'ombre, i cui pericoli sono stati provati sia dal destinatario, Alessandro Rufino, sia dal poeta (vv.7-II), entrambi privati della libertà verosimilmente a causa del servizio cortigiano. Per sopravivere a queste difficoltà bisogna pensare agli splendor celesti, alla virtù e al bene immutabile. La massima morale è disposta a tenaglia ( $\mathrm{vv}$. $5-7$ e vv. I2-I $)$; al centro rimangono le esperienze biografiche dei due protagonisti, simili nella sostanza e, pertanto, espresse con sintassi parallela: tu $v .7 /$ io $v .9$, mentre $v .7 /$ mentre $v .9$, Giacesti $v .9$ / gridai $v . I I$, infermo $v .9$ / salute $v . I I$. 
[65 (CXIX)] Teolo gentil, s'al ver dritto si mira. Il sonetto 65 è indirizzato a un ignoto Teolo, giovane che muove i primi passi (si prolunga la metafora di 64, I-2) nella vita da adulto: a lui il poeta anziiano, che rimpiange gli anni mal spesi ${ }^{42}$ nella ricerca della gloria e il poco tempo superstite, ora che, sconfitti $i$ desideri indegni, la via della virtù è stata finalmente raggiunta, chiede d'essere ricordato negli anni a venire. Come in 64, la disposizione della materia è a tenaglia: la prima quartina e la seconda terzina sono riservate al destinatario, la zona centrale al poeta.

[66 (СиIII) $]$ Sia tanto lungi il tuo focile et l'esca. L'amore, estraneo al trittico di corrispondenza 63-65, pareva esser stato superato a partire da $62 e$ invece incongruamente ricompare per l'ultima volta in 66, capace d'illuminare le menti e dispensare gloria immortale: il poeta, adducendo la debolezza dell'età, prega Amore d'essere liberato dalla sofferenza, ma non dalla spinta nobilitante verso la virtù che gli occhi dell'amata suscitano. Quando nei testi successivi torneranno accenni all'amore, sarà sempre considerato un disvalore da cui liberarsi. Nella prima quartina trova posto la pars destruens della preghiera, cio che il poeta non desidera; nella seconda la sua motivazione, costruita su una sintassi parallela Potei $v$. , / Non posso v. 7; nelle terzine, invece, è la pars construens, ciò che il poeta desidera: anche qui secondo una sintassi binaria che si spartisce con esattezza i sei versi Non cheggio $v .9$ / Ma ch'io possa $v . I 2$.

Tanturli individua tangenze, e Carrai le conferma, con la canzone XXXII di Della Casa, ${ }^{43}$ ma non si vedono rimandi significativi a parte il tema dell'amore in nomo canuto, che è comunque trattato in modo differente: per esempio ofr. vv. 45-46 [Amore] Rendimi il vigor mio [...] / [...] et quella antica forza e v. 5 Libero farmi il tuo fora e 'l mio meglio. Né meglio possono essere accostati $i$ sonetti d'analogo tema di Bembo XCV e XCVI.

[67 (c)] Se 'l tempo fugge et se ne porta gli anni. Nel sonetto 67 il poeta esorta la propria mente (nella seconda parte del sonetto, vv. 7-I4, si susseguono gli imperativi) a prevedere la dannazione futura, a cessare d'affannarsi per l'onore e l'amore, a rivolgersi al cielo (cfr. 64, dove però il discorso è meno evidentemente religioso). Sono da notare i vv. 9-II in cui si riconosce il valore della bellezza terrena, che va lodata ma va anche superata in una prospettiva escatologica. Il sentirsi pellegrino sulla terra, ${ }^{44}$ e quindi fondamentalmente straniero, non preclude l'apprezzamento per il sensibile, che è anch'esso parto di Dio e, come tale, buono e giusto. La svalutazione del corpo e del terreno, che è tanta parte delle rime cinquecentesche (su base petrarchesca, d'ascendenza platonica cooptata al cristianesimo), è un problema di cui non conosco altre soluzioni in poesia. 


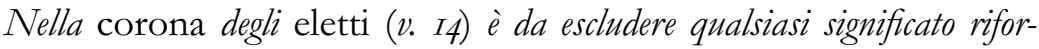
mato, ma l'espressione con ogni probabilità rimanda a un ambiente fervido d'inquietudini e riflessioni religiose, tra cui si possono annoverare Vittoria Colonna (si vedano, infatti, le diverse occorrenze di eletto nelle sue rime), Gasparo Contarini, Reginaldo Pole e quanti furono variamente a favore della riforma cattolica e affascinati da Bernardino Ochino prima che fuggisse oltre frontiera. Guidiccioni nelle sue scritture si attesta su una linea ben più arretrata rispetto ai personaggi ricordati, forse più per la prudenza che gli è propria che per una effettiva distinzione di pensiero.

[68 (CXXV) ] A questa fonte, a cui d'intorno fanno. Il sonetto 68 si lega al precedente grazie a precisi rimandi: le iniuste brame $v .4$ richiamano fero error 67, 5 e false larve 67, 8; l'alma del suo fin presaga $v .13$ ricorda [Mente mia] Non antivedi i tuoi futuri danni 67, 3-4. E un testo di serenità esistenziale (cui si conforma anche la sintassi) provocata dall' ambientazione bucolica (Fossombrone) e dalla solitudine, che permette gli studi, l'indagine socratica e agostiniana di sé (Spio lo mio interno v. 7), la sincerità. Tutto il contrario accade nella corte papale, in cui il poeta entrò undici anni prima: regno delle brame (v. 4), dell'odio e dell'inganno (v. s), dell'invidia ( $v . I 0)$, della dissimulazione ( $v . I I)$, della servitù $(v . I 4)$. Un simile elenco di vizi cortigiani era già nella satira Veggio 'l mio Campo rilevar le ciglia CXXXII, 220-237, databile fra I525 e I528. Il sonetto può essere confrontato con Della Casa LXI, I-6 «De là, dove per ostro et pompa et oro / Fra genti inermi ha perigliosa guerra, / Fuggo io mendico et solo, et di quella esca, / Ch'i' bramai tanto, satio, a queste querce / Ricorro, vago bomai di miglior cibo, / Per haver posa almen questi ultimi annis; il v. rimanda all'abbazia di Nervesa sul Montello, che gioca un ruolo simile a quello di Fossombrone.

[69 (CXX)] Per me da questo mio romito monte. I versi di 69 intrecciano un amore lontano nel tempo, troncato dalla morte che viene ora commemorata con la creazione d'un ruscello artificiale circondato di lauri (difficilmente, mi pare, se ne potrebbe mettere in discussione la verità biografica), e l'amicizia verso il buon Caro, la cui fama è, in quel giro d'anni, più l'augurio d'un ottimo profeta che una presa d'atto. Il sonetto è legato al precedente dalla simile cornice bucolica, pur nella distinzione geografica (Fossombrone in 68, Carignano nei pressi di Lucca in 69), da riprese lessicali (fonte 68, I / fonte v. 4; herbe 68, 2 / pratello v. 3; Qui 68, 5 / Qui di v. 5) e, stilisticamente, dalla sintassi priva di complicażioni.

[70 (CII) ] Traggeti a più bel rio l'ardente sete. Molto raffinato, se non si sovra-interpreta, il passaggio fra 6o e 70 : un ruscello reale $(69$, II) diventa rio 
metaforico $(v . I)$. L'anima, cieca del suo futuro, affonda nel Lethe: non il fume dantesco che prelude all'ascesa al Paradiso, ma quello classico infero, le cui acque costringono a obliare le vere [forme] $e$ lo stato gentile $(62, I I)$. La sete di verità stabili spinge l'anima a elevarsi a più bel rio $e$ a frequentare i luoghi celesti, dove non hanno potere né morte né fortuna, dove i valori terreni sono vani, dove non esiste l'amore terreno, che tiene prigioniero il cuore. Ancora una volta la sintassi si spartisce asimmetricamente il sonetto, secondo lo schema $3+I$. Il primo periodo (vv. I-II) si sviluppa a cascata a partire dai due verbi principali Traggeti $v$. I e ti diporta $v$. s; da quest'ultimo si espandono due ablativi assoluti paralleli, dal secondo dei quali dipende la serie anaforica di Dove ai vv. 9-II.

[7I (CIV)] Duo lustri ho pianto il mio foco vivace. I due testi successivi sembrano formare un dittico, ma a legami deboli, rivolto a Dio. Nel primo, modellato su Rvf. 364, il computo cronologico riassuntivo della vicenda amorosa, ormai conclusa, si accompagna all'invocazione a Dio: solo la sua pietà, sempre superiore ai peccati umani, può purificare le colpe, cosi che il poeta renda degnamente grazie per $i$ doni ricevuti e sciupati. L'amore ba connotati solamente negativi, del tutto incompatibili con una vita pia, visto che dissipa sia il cuore sia anche, più profondamente, l'anima. Lo strale, che in 61 produceva una dolce piaga vitale, qui è avvelenato e causa languore, vaneggiamento e una regressione allo stato ferino. I dieci anni di durata della storia amorosa non forniscono un'indicazione assoluta, perché non ne è stato precisato l'inizio esatto: probabilmente sono da intendere in senso simbolico e non da rapportare con gli undici anni di servizzio presso la corte papale cui si accenna in 68. La zona centrale del sonetto è occupata da un unico periodo ipotetico, in cui la protasi e l'apodosi, ben adagiate nelle partizioni metriche, si contengono rispettivamente nei $v v$. 5-8 e 9-II: in entrambe sono le proposizioni relative ad ampliare la sintassi, che però rimane, qui come nei restanti versi, priva di complicazioni.

[72 (ХСИЛI)] Apra et dissolva il tuo beato lampo. A garantire la transizione e l'unità provvedono le invocazioni Signor verace $7 I$, 5 e sol di gratie v. 2. Nonostante la durezza con cui è stigmatizzato l'amore in $7 I$, nonostante il pentimento del poeta e il suo fervore spirituale, gli occhi della donna riaccendono sempre la tentazione: viene, pertanto, rinnovata la richiesta d'aiuto (cfr. 7I, 5-7 Se la tua santa man [...] non svelle) senza il quale gli sforzi dell'uomo paiono insufficienti a disperdere le nebbie, le insidie e le forze che impediscono di raggiungere la salvezza. ${ }^{45}$ Il velo della materia amorosa non deve coprire un'idea religiosamente pericolosa. Lo sviluppo del periodo nella zona centrale del sonetto è simile a quello di 7 I: la subordinata conces- 
siva, articolata su tre verbi coordinati per polisindeto (avampo $v$. 5, verso $v$. 6, accampo v. 8), occupa i vv. 5-8; la reggente si sviluppa ai vv. 9-II.

[73 ( $C X X I)]$ O messaggier di Dio, che 'n bigia vesta. La luce che sgombra le tempeste $(72, I-2)$ ritorna in 73 associata alla figura del predicatore Bernardino Ochino (la cui fuga nella Gineura di Calvino è posteriore alla morte di Guidiccioni) ${ }^{46}$ Il sonetto forma con il successivo un dittico in lode del predicatore; ad essi si collega 75, in cui il dicitor celeste che con le sue parole dà forza alle virtù teologali va identificato, per forza di macrotesto, nel medesimo Ochino: si noti come 73 e 74 sono indirizzati a un tu, mentre in 75 l'allusione è in terza persona.

In 73 il poeta afferma che gli unici conforti nei tentativi di liberarsi dai legami con il senso famelico sono costituiti non dagli esempi e dalle parole della curia papale, ma dalle prediche contro le ricchezze e le ambizioni pronunciate dal frate.

[74 (CXXII)] A quei ferventi spirti, a le parole. Numerosi sono i legami interni al dittico: sermon santo 73, 3 e detti 73, 7 diventano ferventi spirti v. I, parole v. I e voci v. 8; messaggier di Dio 73, I equivale a Servo fedel di Dio v. 7; lume 73, 5 ritorna in rai v. 9. Come fossero aria i pensieri del poeta, riscaldati dalle parole predicate, salgono a Dio, in una sorta di mistico excessus mentis che permette la visione delle verità paradisiache.

[75 (CXXIII)] O sante figlie de l'eterno Sire. Rivolgendosi in 75 alle tre virtù teologali, il poeta annuncia finalmente il loro completo trionfo, grazie alle parole di Ochino (dicitor celeste v.9), e il pentimento per i peccati compiuti contro di loro. La struttura è un anello ben calibrato: core $v .3$ corrisponde a cor v. I3, folle ardire $v .4$ ad ardì sovrechio $v$. I3; gli assalti $v$. 3 furono rintuzzati dalle repulse $v$. I2; sarete / Vittorïose $v v$. 3-4 è replicato in v'inchina v. I4. La sintassi, come nei due testi precedenti, non è molto articolata. Nella seconda quartina l'anafora già $v .5$ / Già $v .7$ delinea una divisione in distici, in cui si rileva il chiasmo tra v. 5 e v. 8: le due proposizioni (Fuggesi $v .5 \mathrm{e}$ si fa sentire $v .8$ ) sono consecutive rette dalle due coordinate che si spartiscono la prima terzina, in cui si trova il doppio antecedente sì $v v$. g e 10 .

[77 (CXXVI)] Il verde de l'età nel foco vissi. L'ultimo sonetto ricapitola la vicenda biografica narrata. L'amore e il desiderio d'onori, suddivisi equamente nella prima quartina, vengono limitati alla giovinezza; l'età matura si dice spesa nella vana ricerca della gloria poetica (ambizione chiaramente 
denunciata in 50, 7-II), in netta distinzione dalla fama oscura v. 6, che invece è stata conseguita. La seconda terżina risolve in una prospettiva ultraterrena che tutto annulla in sé. Il testo, che nella prima parte procede per distici, nella seconda (vv. 7-I4) è costruito su un unico periodo elaborato, divisibile in due blocchi, vv. 7-II e I2-I4, collegati per opposizione.

Come già rilevato da Tanturli, il testo si può utilmente accostare a Della Casa ХLVII: ma più sono le differenze che le somiglianze. Innanzitutto, la forma metrica: da una parte la discorsività ampia ed elaborata dei II2 versi strutturati in canzone, dall'altra la compressione inevitabile dei I4 versi. Questa netta distinzione retorica fa si che non esistano riscontri testuali d'una qualche cogenza. Nel macrotesto di Della Casa il componimento funge da perno attorno a cui il poeta svolta dagli idoli giovanili verso una maturità di consapevolezza esistenziale e religiosa, ancora incipiente e mal certa: si dubita, infatti, che il penser infermo et lento (la nuova inclinazione verso Dio $v$. I06) sappia la folta / Nebbia cacciare vv. 108-109. In Guidiccioni il sonetto è, al contrario, conclusivo, le parole di Dio sono perentorie: Della Casa primo scrive Non già ch'io scorga il dolce albergo [la morte] anchora (V. I 5$)$, il lucchese già morte il mio dì nel ghiaccio scrive ( $v . I 2)$.

Si noti anche che la successione degli errori denunciata dai due poeti, similmente tripartita, in Della Casa prevede una climax Amore-poesia-onore; in Guidiccioni, invece, Amore-onore-poesia. E probabilmente lecito interpretare la differenza alla luce delle diverse vicende biografiche: Della Casa è letterato di formazione e ambisce alla politica; Guidiccioni, nato maggiorente, è di formazione politico e ambisce alla poesia; entrambi ritroverebbero in ciò che possedevano imperfettamente (il prestigio politico-sociale il primo, la poesia il secondo) quanto di più alto potesse offrire la vita su un piano solo terreno. Altro testo di Della Casa da richiamare è il sonetto XVII Io che l'età solea viver nel fango, preghiera di pentimento a Dio.

Non è improprio caricare di forza simbolica l'ultimo verso del manoscritto, altro splendor che 'l suo più non m'allume: la luce e l'incendio dell'anima, tanto spesso cercato nello sguardo dell' amata e nella gloria, sono ricondotti al sommo sole che ogni altra cosa raggia.

Emilio Torchio 


\section{Giovanni Guidiccioni}

I. In virtù di questa ipotesi, $i$ testi in seconda e terza posizione, assenti dal manoscritto ma individuabili fra le rime tràdite, sono contrassegnati [2] e [3], e i numeri d'ordine 57 e $76-$ corrispondenti a carte bianche (assegnate dall'autore a testi forse esistenti ma non individuabili) - sono saltati.

2. Cfr. Domenico De Robertis, Commentare la poesia, commentare la prosa, in Il commento ai testi. Atti del seminario di Ascona 2-9 ottobre 1989, a cura di O. Besomi e C. Caruso, BaselBoston-Berlin, Birkhauser Verlag, I992, pp. I69-77, a p. I72: «Si aggiunga l'altro carattere del commento, nel suo accompagnare passo passo il testo: la sua naturale ripetitività, in connessione col riprodursi nel testo di situazioni formali o d'informazione analoghe».

3. All'etichetta «canzoniere» si preferisce questa dicitura tecnica e non elegante perché più neutra e meno compromessa con Petrarca.

4. Non coglie nel segno, quindi, l'ipotesi di Minutoli (Opere di Monsignor Giovanni Guidiccioni, a cura di Carlo Minutoli, Firenze, Barbèra, I 867, p. I03): «Questo sonetto meglio che al sacco dato a Roma dagl'Imperiali nel maggio del i 527 è forse da riferire al fatto dei Colonnesi che nell'anno innanzi per odio a Clemente VII aveano assaltato e messo a ruba il Vaticano e la stessa Basilica di San Pietro. Infatti se il Duca d’Urbino avrebbe potuto punire i Colonnesi ribelli, e vendicare l'oltraggio recato al Pontefice, il che avrebbe anche dovuto come Prefetto di Roma, sarebbe stato un pretendere sopra le sue forze se si fosse voluto ch'egli liberasse Roma dall'esercito di Carlo V, mentre non era che uno dei capitani della Lega, di cui il comando supremo era tenuto dal francese Lautrec: onde non gli sarebbe stato giustamente applicabile l'ultimo verso [...]». Le lettere si citano secondo l'edizione curata da Maria Teresa Graziosi, Roma, Bonacci, I979.

5. Rispettivamente cfr. Guidiccioni, Opere cit., e E. Chiorboli, Giovanni Guidiccioni, Jesi, Stab. tipografico cooperativo, I907, la cui impostazione si ritrova nella successiva edizione per gli «Scrittori d'Italia».

6. Dall'edizione a cura di Carlo Dionisotti, Pietro Bembo, Prose e rime, Torino, Utet, I $996^{2}$, si cita il numero d'ordine in cifra arabica; dall'edizione a cura di Guglielmo Gorni, in Poeti del Cinquecento. Tomo I. Poeti lirici, burleschi satirici e didascalici, a cura di G. Gorni, M. Danzi e S. Longhi, Milano-Napoli, Ricciardi, pp. 39-225, la lettera del testo (che corrisponde all'edizione Venezia, Giovan Antonio e fratelli da Sabbio, I 530 ) e il numero in cifra romana.

7. Gorni, Poeti del Cinquecento cit., pp. 529-30.

8. L'invocazione va confrontata con SANNAZARO 93, I-3 «[Roma] Gloriosa, possente, antica madre, / che nel tuo grembo alberghi uomini e Dei, / di palme un tempo ornata e di trofei» e v. I 2 "[Roma] O del mondo regina, invitta terra» (ed. a cura di Alfredo Mauro, Bari, Laterza, I 96I).

9. Il motivo risale a Genesis I9, 24 «Igitur Dominus pluit super Sodomam et Gomorram sulphur et ignem a Domino de caelo»; il fuoco punitivo è qui trasformato in acqua salvatrice a causa del v. 4; cfr. anche Rvf. I 28, 69-70 «ma 'l vostro sangue piove / più largamente, ch'altr'ira vi sferza»; Rvf. I 36, I «Fiamma dal ciel su le tue treccie piova» e Colonna E2 3, I-2 «Prego il Padre divin che tanta fiamma / mandi del foco Suo nel vostro core, / Padre nostro terren, che de l'ardore / de l'ira umana in voi non resti dramma». Analogamente viene invocato l'intervento divino a 7 . I e I 3. 9-I 2.

ı. Per il motivo cfr. Bembo C (го9), I-4 «La nostra e di Iesù nemica gente, / [...] / [...] in parte ha l'odio scarco / Sovra quei, che la fer già sì dolente»: il sonetto è ricordato in Lettere I. 


\section{Emilio Torchio}

I I. Cfr. Molza II son. 37, I-2 «Da gli empi strali, onde riman ferita / Del mondo in guisa la più bella parte» e I son. I 36, 9-1 I «Risani pria le piaghe aspre, e mortali, / C’hanno il bel corpo de l'Italia guasto; / Poi verso l'Oriente spieghi l'ali» (Delle poesie volgari e latine, a cura di Pierantonio Serassi, Bergamo, Lancellotti, 1747-54, 3 voll.).

I 2. Per l'iconografia tradizionale della «Pace bianca il seno e 'l volto / Et la man carca di mature spiche» vv. 8-9 cfr. Tiвullo I го, 67-68 «at nobis, Pax alma, veni spicamque teneto, / profluat et pomis candidus ante sinus» e CAPpello son. 306, I-3 «Di ricche spiche cinta, e di feconde / Viti ornata le tempie, a noi sen viene / La santa Pace» (ed. a cura di Pierantonio Serassi e Agamiro Pelopideo, Bergamo, Lancillotti, 1753).

I 3. Il testo si può confrontare con MoLzA II son. I4, I-2 «Quando fia mai ch'i nostri dolci campi / Lunga pace, Signori, riveggia, ed orni? / E chiari come già tornino i giorni, / Ne' qua' di bei desir ciascun avvampi? / Chi verrà, che dal giogo aspro ne scampi, / E lieta faccia a le campagne torni? / Sicché 'l barbaro folle se ne scorni, / Né trovi schermo al nostro ferro, o scampi?». La formula introduttiva è piuttosto diffusa a partire da $R v f .253,2$ «or fia mai il dì ch'i' vi riveggia et oda?».

I4. Il testo va confrontato con Minturno, Rime et prose, Venezia, Rampazzetto, i 559 , p. I 30 «Re degli altri, superbo, invitto augello, / Che porti l'arme a Giove e 'n ciel t'annidi, / Mentre di morte il Gallo altero sfidi / Irato e turbi la magion di quello, / Non vedi com'al sol ne' tracii lidi / Vibra la lingua e si fa novo e bello / Inteso a danni de' tuoi cari nidi / Il nemico serpente a Dio rubello. / Spiega quivi il gran volo, ivi gli artigli / Tingi ne l'empio sangue di quel monstro [...]»; con ColonNA E25, I-8 «Sento per gran timor con alto grido / al venir d'una excelsa Aquila altera / fuggir tutti gli augelli in varia schiera, / né pur fidarsi ancor nel proprio nido. / Ella sicura, col presidio fido / de' cieli e de la sua virtù sincera, / per novo onor con maggior gloria spera / volar superba in ogni extremo lido» (ed. a cura di Alan Bullock, Bari, Laterza, I982); e con il testo che Bullock, Vittoria Colonna: note aggiunte alla edizione critica del 1982, in "Giornale Storico della Letteratura Italiana», I62 (1985), pp. 407-19, a p. 408, pubblica dal ms. B V 5 della Biblioteca Jacobilli di Foligno, rifiutandone l'attribuzione alla Colonna: «Hor ti desta veloce, Augel di Giove, / Contra l'hostile et foribondo ardire / Qual pur sfogando i primi orgogli e l'ire / Fallace man alli tuoi danni move. / Dal ciel ogn'hor nelle tue arme piove / Giusta cagion, così giusto martire / Pronto riveggio a quelle inique e dire / Barbare voglie audaci et empie prove» (vv. I-8).

I 5 . La sottrazione dei frutti da parte degli invasori rimanda a VirgiLIO, Buc. I, 70-73 «Impius haec tam culta novalia miles habebit? / Barbarus has segetes [...] His nos consevimus agros!). Si noti anche una più ampia somiglianza: come nella prima ecloga Melibeo vede finire i propri campi in preda a soldati stranieri, così il passaggio delle truppe germaniche portava con sé saccheggi e violenza.

I6. I versi vanno confrontati con MoLzA II son. 31, 3-4 «Di pietà gli occhi, a chi ti chiama, e inchina, / Non negar prego da giusta ira accesa» e MolzA I son. 3 I , 9- I 4 «E se giust'ira a vendicar t'invia / Le nostre colpe $[\ldots] /[. .$.$] E quivi spendi ogni tuo irato strale».$

17. Cfr., per contrasto, i vv. I 2-I 3 «Qui i vomeri et le falci in via più crudi / Ferri converse» con Michea 4, 3 «et iudicabit inter populos multos et corripiet gentes fortes usque in longinquum et concident gladios suos in vomeres et hastas suas in ligones, non sumet gens adversus gentem gladium et non discent ultra belligerare».

I8. Cfr. Molza II son. io, 7-9 «Qui sul Tevere, u' teco ora dimoro, / Sol duri affanni n'impromesse Marte. / Qui svelti i lauri sono, e de le olive / Translato in tutto il chiaro germe altrove».

ig. Giovanni Della Casa, Rime, a cura di Giuliano Tanturli, Parma, Fondazione Pietro Bembo / Ugo Guanda Editore, 200I, pp. 2 I e 22. 


\section{Giovanni Guidiccioni}

20. Guidiccioni, Opere, p. I07.

2i. Bernardo Tasso, Rime, vol. II, a cura di Vercingetorige Martignone, Torino, Res, i 995.

22. I rapporti «di amicizia e solidarietà letteraria che la Colonna ebbe col Guidiccioni [...] richiederebbero lungo discorso» (Carlo Dionisotti, Appunti sul Bembo e su Vittoria Colonna, in Miscellanea Augusto Campana, Padova, Antenore, I98 I, pp. 257-86, a p. 268).

23. Per i legami al presente contesto cfr. Colonna Ar: 2 I, I, 5-8 «Questo sol, ch'oggi agli occhi splende / perché con l'altro mio più non contende, / ch'or lampeggiando nel divin soggiorno / d'un ardor santo e d'un perpetuo giorno / dinanzi al vero Sol s'alluma e accende»; E24, I-2 «Nel mio bel Sol la vostra Aquila altera / fermò già gli occhi» e 5-6 «Or che la chiara luce alma e sincera / oscura nube le nasconde e vieta».

24. Presente già nella fonte petrarchesca $R v f$. 299, I 3-I4 «Quanto al misero mondo, et quanto manca [: l'alma stanca] / agli occhi miei».

25. Per i vv. I-4 cfr. Colonna A2: i 5, 5-6 «[m’involo] perché expedito al Sol ch'adoro e colo / vada il pensiero», A2: I 3 . 5 «S'erge pensier col sol, ond'io ritorno / al mio», Aı: 60, I-4 «Quando io son tutta col pensier rivolta / ai raggi e al caldo del mio vivo Sole, / a quelle chiare luci ardenti e sole / ch'apparver qui fra noi sol una volta, / l'alma vede la sua sì bella, e ascolta / sì vere le divine alte parole» e AI: 4I, I-6 «Alzata al Ciel da quel solingo e raro / pensier che sovra il corso uman mi spinge / veder mi parve il volto che dipinge / Amor al cor, ma più splendente e chiaro; / e udir su questi cerchi or lieto [forse da correggere lieta] imparo / come un solo voler li move e cinge»; per il v. 6 cfr. A2: 38, 5-6 «e là su ne la sua [del marito] divina scola / [l'alma] impara cose»; per il v. I 2 cfr. A2: I 5 , I 2-I 4 «Ma se potesse l'alta sua sembianza / formar quant'ella vuol l'accesa mente / parte avrei forse qui del ben perfetto».

26. Cfr. $R v f$. 349, 9-1 4 «O felice quel dì che, del terreno / carcere uscendo, lasci rotta et sparta / questa mia grave et frale et mortal gonna, / et da sì folte tenebre mi parta / volando tanto su nel bel sereno, / ch'i’ veggia il mio Signore et la mia donna».

27. Il primitivo incipit era Anima bella, il cui candido velo. Se la correzione bella > eletta pare opportuna dato il contesto funebre, quella candido > leggiadro riesce a prima vista più infelice: il candore del corpo, cioè la sua purezza, aggiunge spiritualità all'amore. Forse la variante fu messa in opera per evitare la ripetizione concettuale con casti v. 4; o forse perché la castità della donna è marca precipua del secondo amore narrato dal macrotesto: la correzione vorrebbe così tenere distinte le caratteristiche delle due donne.

28. Per il v. 7 cfr. Rvf. 360 , I 49-50 «- Ben me la die', ma tosto la ritolse. - / Responde: - Io no, ma Chi per sé la volse». Da notare lo schema sintattico inusuale ${ }_{1}+{ }_{2}+\mathrm{I}$, come anche il ritmo binario: Se v. 3 / se v. 4, occhi v. 3 / beltà v. 4, luce / speme v. 5, Morte v. 6 / Chi v. 7, spense v. 6 / ritolse v. 7, scerneva v. Io / chiudeva v. I I, lentato v. I 2 / scosso v. I 3, lieto / beato v. I4.

29. Il sonetto di Tolomer edito in De le rime di diversi nobili poeti toscani, raccolte da $\mathrm{m}$. Dionigi Atanagi, libro secondo, Venezia, Avanzo, i 565 , c. I $8 v$, ha somiglianze significative col presente testo e si potrebbe supporre, con ogni cautela, che sia indirizzato a Guidiccioni: «I vostri alti pensier di virtù pieni / Saglion là dov’è il ciel fermo e lucente: / Quivi accesi da Dio di grazia ardente, / Tornano a voi più caldi e più sereni. / Voi per questi egri, poi, campi terreni / Gli spargete a sanar la losca gente, / Ch'aprendo gli occhi a un falso ben presente, / Gli chiude al sol de' veri eterni beni. / Ben ha lo spirto vil, la carne grave / Chi non ode, non vede e pensa seco / I detti e l'opre e vostra alta virtute, / Che i belli essempi e l'harmonia soave / E la mente divina al mondo cieco / E 'nfermo porge ognor lume e salute». 


\section{Emilio Torchio}

30. Per il v. I «Per vestirne virtù che nuda giace» cfr. DanTe, Se vedi gli occhi miei, vv. I 2-I 3 «Questa vertù che nuda e fredda giace, / Levala su vestita de 'l tuo velo» (Sonetti e canzoni di diversi antichi autori toscani, Firenze, eredi di Filippo di Giunta, I 527 , 1. II c. I 8 r). Preciso anche il ricordo petrarchesco per i vv. I 2-I 3 《il suo cor che non consente / Al tuo morir»: Rvf. I4I, I4 «et cieca al suo morir l'alma consente».

3r. La formula di passaggio ha attestazioni in Bembo, Cappello, Tarsia.

32. La maiuscola è nel manoscritto ed è conservata proprio nell'ipotesi che renda più esplicito il gioco onomastico. La minuscola, peraltro, non sposterebbe la questione.

33. Cfr. Colonna Ai: 54, 9-I « «'ivi [in cielo, sogg: l’alma] s'appaga, si nudrisce e vive, / e l'abitare in questo carcer sempre / le saria grave». Per la formulazione verbale cfr. anche Rvf. I 40, I-2 «Amor, che nel penser mio vive et regna [: insegna: sdegna]/ e 'l suo seggio maggior nel mio cor tene, / talor armato ne la fronte vène: / ivi si loca».

34. Alla ovvia fonte petrarchesca (Rvf. 366, I I I-I 2$)$ si può allegare il passo parallelo, in cui vige analogia di situazione (l'anima dell'amante presso l'amata), CAppello son. 82, I 2 «rimarrei sasso che spargesse umore».

35. Cfr. Molza I son. 8 I, I-3 «Come stella, che fuor dell'Oceano / Per far al Ciel di ricco fregio onore, / Esce dinanzi al matutino albore» e I son. I 89. 3-4 «E schernendo del mondo ogni pensiero / Scarche poggiate al Cielo, e peregrine».

36. Per la struttura sintattica «Chi desia di veder [...] Venga» cfr. Rvf. 248, I-2 «Chi vuol veder quantunque pò Natura / e 'l Ciel tra noi, venga a mirar costei», Alamanni, Opere toscane, vol. I, p. 202 «Chi desia di veder più bella Luna /[...]/ Venga questa a mirar», Colonna Si: I09, I-5 «Chi desia di veder $[\ldots .] /.[. .$.$] miri la Vergin sacra», SANnAZARo 80, I-3$ «Chi vòl meco piangendo esser felice $/[. .$.$] / venga a veder questa».$

37. Gorni, Poeti del Cinquecento cit., p. 549.

38. I versi non sono chiarissimi, ma la donna, rivolgendosi al suo Signor, dichiara che il proprio cuore è trafitto dallo strale di lui: non sembrando probabile, visto il contesto mitologico, che Signor sia 'Dio', si deve pensare sia l'amante.

39. Alternativamente, è comunque lecito supporre un macrotesto debole immaginando che la donna si trovi già a Firenze e il poeta inviti a far festa mentre lei appare. La situazione sarebbe, quindi, analoga a quella descritta nel cavalcantiano Chi è questa che ven, in cui la donna non giunge da lontano, ma semplicemente compare inaspettata.

40. Cfr. Colonna Si: 27 «Se 'l breve suon che sol quest'aer frale / circonda e move, e l'aura che raccoglie / lo spirto dentro, e poi l'apre e discioglie / soavemente in voce egra e mortale, / con tal dolcezza il cor sovente assale / che d'ogni cura vil s'erge e ritoglie, / sprona accende 'l pensier, drizza le voglie / per gir volando al Ciel con leggiere ale, / che fia quand'udirà con vivo zelo / la celeste armonia l'anima pura / sol con l'orecchia interna intenta al vero / dinanzi al suo fattor nel sommo Cielo, / u' non si perde mai tuono o misura, / né si discorda il concento altero?». Di tema prossimo, ma meno pertinente Si: 28.

4I. La contrapposizione tra forme visibili e forme vere è qui di un platonismo fortemente cristianizzato, a differenza, ad esempio, che in Asolani III is «voi ciechi, d'intorno a quelle vostre false bellezze, a guisa di Narciso vi pascete di vano disio, e non v'accorgete che elle sono ombre della vera, che voi abandonate».

42. Cfr. $R v f$. 364, IO-I I «pentito et tristo de’ miei sì spesi anni, / che spender si deveano in miglior uso». 


\section{Giovanni Guidiccioni}

43. Rispettivamente nell'ed. cit. pp. 77-78 e in Giovanni Della Casa, Rime, a cura di Stefano Carrai, Torino, Einaudi, 2003, p. 90.

44. Cfr. Colonna Si: I 58 , I-I I «Oh quanto il nostro infermo lume appanna / la nebbia rea delle speranze insane! / [...] l'uom si strugge e affanna / in cercar le ricchezze e glorie umane, / fermando l'occhio in queste luci vane, / col suo proprio desir sé stesso inganna. / Convien qual peregrin sciolto e leggiero, / gir con l'opre amorose e con la mente / fidele e salda al glorioso albergo».

45. Cfr. Colonna, Si: i9, i-8 «Anima, il Signor viene! omai disgombra / le folte nebbie intorno dal tuo core / acciò che l'ugge del terreno amore / a l'alta luce Sua non faccian ombra! / E perché il fallir nostro spesso ingombra / la vista, sì ch’a quel chiaro splendore / passar non può, da te scaccia l'errore / ch'agli occhi tuoi cotanto ben adombralı.

46. Per i rapporti del frate con gli ambienti letterari cfr. Giovanni Bardazzi, Le rime spirituali di Vittoria Colonna e Bernardino Ocbino, in «Italique», 4 (200I), pp. 6I-IoI. 